\title{
EMPIRICAL LIKELIHOOD BASED CONFIDENCE REGIONS FOR FIRST ORDER PARAMETERS OF HEAVY-TAILED DISTRIBUTIONS
}

\author{
Julien Worms(1) \& Rym Worms (2) \\ (1) Université de Versailles-Saint-Quantin-En-Yvelines \\ Laboratoire de Mathématiques de Versailles (CNRS UMR 8100), \\ UFR de Sciences, Bât. Fermat, \\ 45 av. des Etats-Unis, 78035 Versailles Cedex, \\ e-mail : worms@math.uvsq.fr \\ (2) Université Paris-Est-Créteil \\ Laboratoire d'Analyse et de Mathématiques Appliquées (CNRS UMR \\ 8050), \\ 61 av. du Général de Gaulle, 94010 Créteil cedex, \\ e-mail : rym.worms@u-pec.fr
}

AMS Classification. Primary 62G32 ; Secondary 62G15.

Keywords and phrases. Extreme values. Generalized Pareto Distribution. Confidence regions. Empirical Likelihood. Profile empirical likelihood.

\begin{abstract}
Let $X_{1}, \ldots, X_{n}$ be some i.i.d. observations from a heavy tailed distribution $F$, i.e. such that the common distribution of the excesses over a high threshold $u_{n}$ can be approximated by a Generalized Pareto Distribution $G_{\gamma, \sigma_{n}}$ with $\gamma>0$. This paper deals with the problem of finding confidence regions for the couple $\left(\gamma, \sigma_{n}\right)$ : combining the empirical likelihood methodology with estimation equations (close but not identical to the likelihood equations) introduced by J. Zhang (2007), asymptotically valid confidence regions for $\left(\gamma, \sigma_{n}\right)$ are obtained and proved to perform better than Wald-type confidence regions (especially those derived from the asymptotic normality of the maximum likelihood estimators). By profiling out the scale parameter, confidence intervals for the tail index are also derived.
\end{abstract}

\section{Introduction}

In statistical extreme value theory, one is often interested by the estimation of the socalled tail index $\gamma=\gamma(F)$ of the underlying model $F$ of some i.i.d. sample $\left(X_{1}, \ldots, X_{n}\right)$, which is the shape parameter of the Generalized Pareto Distribution (GPD) with distribution function (d.f.)

$$
G_{\gamma, \sigma}(x)=\left\{\begin{array}{cc}
1-\left(1+\frac{\gamma x}{\sigma}\right)^{-\frac{1}{\gamma}}, & \text { for } \gamma \neq 0 \\
1-\exp \left(-\frac{x}{\sigma}\right), & \text { for } \gamma=0 \\
1 &
\end{array}\right.
$$


The GPD appears as the limiting d.f. of excesses over a high threshold $u$ defined for $x \geq 0$ by

$$
F_{u}(x):=\mathbb{P}(X-u \leq x \mid X>u) \text {, where } X \text { has d.f. } F .
$$

It was established in J. Pickands (1975) and A. Balkema and L. de Haan (1974) that $F$ is in the domain of attraction of an extreme value distribution with shape parameter $\gamma$ if and only if

$$
\lim _{u \rightarrow s_{+}(F)} \sup _{0<x<s_{+}(F)-u}\left|F_{u}(x)-G_{\gamma, \sigma(u)}(x)\right|=0
$$

for some positive scaling function $\sigma(\cdot)$, where $s_{+}(F)=\sup \{x: F(x)<1\}$. This suggests to model the d.f. of excesses over a high threshold by a GPD. This is the P.O.T method.

Some estimation methods for the couple $(\gamma, \sigma)$ in the GPD parametrization have been proposed. We can cite the maximum likelihood (ML) estimators of R. L. Smith (1987) or the probability weighted moments (PWM) estimators of J. Hosking and J. Wallis (1987). In J. Zhang (2007), the author proposed new estimators based on estimating equations close to the likelihood equations. Using the reparametrization $b=-\gamma / \sigma$ and considering $X_{1}, \ldots, X_{n}$ i.i.d. variables with distribution $G_{\gamma, \sigma}$ with $\sigma$ a fixed value (which is an important restriction if the aim is to prove asymptotic results), he based his method on one of the likelihood equations

$$
\gamma=-\frac{1}{n} \sum_{i=1}^{n} \log \left(1-b X_{i}\right)
$$

and on the empirical version of the moment equation $\mathbb{E}\left(\left(1-b X_{1}\right)^{r}\right)=\frac{1}{1-r \gamma}$, i.e.

or

$$
\frac{1}{n} \sum_{i=1}^{n}\left(1-b X_{i}\right)^{r}-\frac{1}{1-r \gamma}=0
$$

$$
\frac{1}{n} \sum_{i=1}^{n}\left(1-b X_{i}\right)^{r / \gamma}-\frac{1}{1-r}=0
$$

for some parameter $r<1$. (2) and (3) yield the estimation equation for $b$

$$
\frac{1}{n} \sum_{i=1}^{n}\left(1-b X_{i}\right)^{n r\left(\sum_{i=1}^{n} \log \left(1-b X_{i}\right)\right)^{-1}}-\frac{1}{1-r}=0, \quad \text { provided } b<X_{(n)}^{-1} \text { and } r<1 .
$$

An estimation of $\gamma$ is then deduced from (2) and $\sigma$ is estimated using $b=-\gamma / \sigma$.

Zhang proved in J. Zhang (2007) (Theorem 2.2) that for a $\operatorname{GPD}(\gamma, \sigma)$ sample with $\gamma>-1 / 2$, the estimators he proposed for $\gamma$ and $\sigma$ are jointly asymptotically normally distributed and that they share none of the following drawbacks of the ML and PWM methods : theoretical invalidity of large sample results for the PWM estimators with large positive $\gamma$, and computational problems for the ML estimator.

In this paper, we consider the classical P.O.T. framework, where an i.i.d. sample $X_{1}, \ldots, X_{n}$ with distribution $F$ is observed and, according to (1), a GPD $G_{\gamma, \sigma\left(u_{n}\right)}$ is fitted to the sample of the excesses over a large threshold $u_{n}$. Noting $\sigma_{n}=\sigma\left(u_{n}\right)$, our goal is to build confidence regions for the couple $\left(\gamma, \sigma_{n}\right)$ (as well as confidence intervals 
for the tail index $\gamma$ ) for heavy-tailed distributions ( $\gamma>0$ case), starting from Zhang's estimating equations; therefore the excesses will be approximately GPD distributed and the parameter $\sigma=\sigma_{n}$ will be varying with $n$. To the best of our knowledge, little attention has been paid to the subject of joint confidence regions (and their coverage probabilities) for the couple $\left(\gamma, \sigma_{n}\right)$, especially outside the exact GPD framework.

An obvious approach to obtain confidence regions is to use the gaussian approximation. In this work, we consider an alternative method, namely the empirical likelihood method. This method was developped by Owen in A.B. Owen (1988) and A.B. Owen (1990), for the mean vector of i.i.d. observations and has been extended to a wide range of applications, particularly for generalized estimating equations (Y. S. Qin and J. Lawless (1994)) .

In J.C. Lu and L. Peng (2002), this method was applied to construct confidence intervals for the tail index of a heavy-tailed distribution (empirical likelihood estimator of the tail index being equal to the Hill estimator). It turned out that the empirical likelihood method performs better than the normal approximation method in terms of coverage probabilities especially if the calibration method proposed in L. Peng and Y. Qi (2006) is adopted. We will see that it is even more the case for confidence regions.

In Section 2, we explain the empirical likelihood methodology based on Zhang's equations (2) and (4), and present some asymptotic results. A simulation study is conducted in Section 3, which compares different methods for constructing confidence regions for the couple $\left(\gamma, \sigma_{n}\right)$, as well as confidence intervals for $\gamma$ alone, in terms of coverage probabilities. Proofs are given in Section 4 and some details left in the Appendix. Technical difficulties are mainly due to the fact that one of the parameters and the distribution of the excesses are depending on $n$.

\section{Methodology and statement of the results}

\subsection{Notations and Assumptions}

In this work, the tail index $\gamma$ is supposed to be positive and $F$ twice differentiable with well defined inverse $F^{-1}$. Let $V$ and $A$ be the two functions defined by

$$
U(t)=\bar{F}^{-1}(1 / t) \quad \text { and } \quad A(t)=t \frac{U^{\prime \prime}(t)}{U^{\prime}(t)}+1-\gamma,
$$

where $\bar{F}=1-F$.

We suppose the following first and second order conditions hold $\left(R V_{\rho}\right.$ below stands for the set of regularly varying functions with coefficient of variation $\rho$ ) :

$$
\lim _{t \rightarrow+\infty} A(t)=0
$$

$A$ is of constant sign at $\infty$ and there exists $\rho \leq 0$ such that $|A| \in R V_{\rho}$.

A proof of the following lemma can be found in L. de Haan (1984).

Lemma 1. Under (5) and (6) we have, for all $x>0$,

$$
\left(\frac{U(t x)-U(t)}{t U^{\prime}(t)}-\frac{x^{\gamma}-1}{\gamma}\right) / A(t) \longrightarrow K_{\gamma, \rho}(x) \text {, as } t \rightarrow+\infty,
$$


where $K_{\gamma, \rho}(x):=\int_{1}^{x} u^{\gamma-1} \int_{1}^{u} s^{\rho-1} d s d u$, and the following well-known Potter-type bounds hold:

$\forall \epsilon>0, \exists t_{0}, \forall t \geq t_{0}, \forall x \geq 1$,

$(1-\epsilon) \exp ^{-\epsilon \log (x)} K_{\gamma, \rho}(x) \leq\left(\frac{U(t x)-U(t)}{t U^{\prime}(t)}-\frac{x^{\gamma}-1}{\gamma}\right) / A(t) \leq(1+\epsilon) \exp ^{\epsilon \log (x)} K_{\gamma, \rho}(x)$.

\subsection{Confidence regions for the couple $\left(\gamma, \sigma_{n}\right)$}

For some $r<1$ and positive $y, \gamma, \sigma$, let

$$
g(y, \gamma, \sigma):=\left(\begin{array}{c}
\log (1+\gamma y / \sigma)-\gamma \\
(1+\gamma y / \sigma)^{r / \gamma}-\frac{1}{1-r}
\end{array}\right) .
$$

Note that, if $Z_{1}, \ldots, Z_{n}$ are i.i.d. $\operatorname{GPD}(\gamma, \sigma)$, then $\frac{1}{n} \sum_{i=1}^{n} g\left(Z_{i}, \gamma, \sigma\right)=0$ summarizes equations (2) and (3) of J. Zhang (2007).

Let $X_{1}, \ldots, X_{n}$ be i.i.d. random variables with common d.f. $F$ (satisfying the assumptions stated in the previous paragraph), and $\gamma_{0}$ and $\sigma_{0}(\cdot)$ be the true parameters such that relation (1) is satisfied. For a fixed high threshold $u_{n}$, consider the $N_{n}$ excesses $Y_{1} \ldots, Y_{N_{n}}$ over $u_{n}$. Conditionally on $N_{n}=k_{n}, Y_{1} \ldots, Y_{k_{n}}$ are i.i.d. with common distribution function $F_{u_{n}}$ which , according to (1), is approximately $G_{\gamma_{0}, \sigma_{0 n}}$, where $\sigma_{0 n}:=\sigma_{0}\left(u_{n}\right)$. The objective is to estimate $\gamma_{0}$ and $\sigma_{0 n}$.

Let $\mathcal{S}_{n}$ denote the set of all probability vectors $p=\left(p_{1}, \ldots, p_{k_{n}}\right)$ such that $\sum_{i=1}^{k_{n}} p_{i}=1$ and $p_{i} \geq 0$. The empirical likelihood for $(\gamma, \sigma)$ is defined by

$$
L(\gamma, \sigma):=\sup \left\{\prod_{i=1}^{k_{n}} p_{i} / p \in \mathcal{S}_{n} \text { and } \sum_{i=1}^{k_{n}} p_{i} g\left(Y_{i}, \gamma, \sigma\right)=0\right\}
$$

and the empirical log likelihood ratio is then defined as

$$
l(\gamma, \sigma):=-2\left(\log L(\gamma, \sigma)-\log L\left(\hat{\gamma}_{n}, \hat{\sigma}_{n}\right)\right)
$$

where $\left(\hat{\gamma}_{n}, \hat{\sigma}_{n}\right)$ are maximising $L(\gamma, \sigma)$, and are called the maximum empirical likelihood estimates (MELE) of the true parameters $\gamma_{0}$ and $\sigma_{0 n}$.

Since Theorem 2.1 of J. Zhang (2007) implies that, for $r<1 / 2$, there exists a unique and easily computable solution $\left(\tilde{\gamma}_{n}, \tilde{\sigma}_{n}\right)$ to the equations

$$
\frac{1}{k_{n}} \sum_{i=1}^{k_{n}} \log \left(1+\gamma Y_{i} / \sigma\right)-\gamma=0 \quad \text { and } \frac{1}{k_{n}} \sum_{i=1}^{k_{n}}\left(1+\gamma Y_{i} / \sigma\right)^{r / \gamma}-\frac{1}{1-r}=0
$$

i.e. such that $k_{n}^{-1} \sum_{i=1}^{k_{n}} g\left(Y_{i}, \tilde{\gamma}_{n}, \tilde{\sigma}_{n}\right)=0$, it thus comes that $L\left(\tilde{\gamma}_{n}, \tilde{\sigma}_{n}\right)=k_{n}^{-k_{n}}$ which is equal to $\max _{\gamma, \sigma} L(\gamma, \sigma)$ : the MELE estimators $\left(\hat{\gamma}_{n}, \hat{\sigma}_{n}\right)$ therefore coincides with Zhang's estimators.

Note however that Zhang worked in the purely GPD framework and that the application of his results for constructing confidence regions, based on the asymptotic normality of $\left(\hat{\gamma}_{n}, \hat{\sigma}_{n}\right)$, necessarily involves some additional covariance estimation. Our aim is to construct confidence regions for $\left(\gamma, \sigma_{n}\right)$ directly, relying on the asymptotic distribution of the empirical likelihood ratio $l\left(\gamma_{0}, \sigma_{0 n}\right)$ stated in the following theorem. Classical 
advantages of proceeding so are well known : a first one is the avoidance of information matrix estimation, a second one is the guarantee of having the confidence region included in the parameter space (as a matter of fact, in our case, the parameter $\sigma$ is positive but nothing guarantees that the confidence region for $(\gamma, \sigma)$, based on the CLT for $\left(\hat{\gamma}_{n}, \hat{\sigma}_{n}\right)$ will not contain negative values of $\sigma$ ). Note in addition that our result is proved in the general framework (i.e. when the excess distribution function is supposed to be only approximately GPD) and that simulation results show some improvements in terms of coverage probability (see next Section).

Note that the empirical $\log$-likelihood ratio $l(\gamma, \sigma)=-2 \log \left(k_{n}^{k_{n}} L(\gamma, \sigma)\right)$ has a more explicit expression : following A.B. Owen (1990), the Lagrange multipliers method yields

$p_{i}=\frac{1}{k_{n}\left(1+<\lambda(\gamma, \sigma), g\left(Y_{i}, \gamma, \sigma\right)>\right)} \quad$ and $l(\gamma, \sigma)=2 \sum_{i=1}^{k_{n}} \log \left(1+<\lambda(\gamma, \sigma), g\left(Y_{i}, \gamma, \sigma\right)>\right)$,

where $\lambda(\gamma, \sigma)$ is determined as the solution of the system

$$
\frac{1}{k_{n}} \sum_{i=1}^{k_{n}}\left(1+<\lambda(\gamma, \sigma), g\left(Y_{i}, \gamma, \sigma\right)>\right)^{-1} g\left(Y_{i}, \gamma, \sigma\right)=0
$$

Let $a_{n}:=A\left(1 / \bar{F}\left(u_{n}\right)\right)$.

Theorem 1. Under conditions (5) and (6), with $\gamma>0$, conditionally on $N_{n}=k_{n}$, if we suppose that $k_{n}$ tends to $+\infty$ such that $\sqrt{k_{n}} a_{n}$ goes to 0 as $n \rightarrow+\infty$, then for $r<1 / 2$

$$
l\left(\gamma_{0}, \sigma_{0 n}\right) \stackrel{\mathcal{L}}{\rightarrow} \chi^{2}(2) \text {, as } n \rightarrow+\infty .
$$

This result is the basis for the construction of a confidence region, of asymptotic level $1-\alpha$, for the couple $\left(\gamma_{0}, \sigma_{0 n}\right)$ which consists in all $(\gamma, \sigma)$ values such that $l(\gamma, \sigma) \leq c_{\alpha}$, where $c_{\alpha}$ is the $1-\alpha$ quantile of the $\chi^{2}(2)$ distribution.

Note that $\sqrt{k_{n}} a_{n} \rightarrow 0$ was also assumed in J.C. Lu and L. Peng (2002).

\subsection{Confidence interval for $\gamma$}

For a fixed parameter $\gamma$, we note $\hat{\sigma}_{\gamma}$ the value of $\sigma$ that minimizes $l(\gamma, \sigma)$. Then, $l\left(\gamma, \hat{\sigma}_{\gamma}\right)$ is called the profile empirical log likelihood ratio. The following asymptotical result is the basis for constructing the confidence intervals for the true parameter $\gamma_{0}$ of the model.

Theorem 2. Under the same conditions as Theorem 1 , if $r<1 / 3$ then, conditionnally on $N_{n}=k_{n}$,

$$
l\left(\gamma_{0}, \hat{\sigma}_{\gamma_{0}}\right) \stackrel{\mathcal{L}}{\rightarrow} \chi^{2}(1) \text {, as } n \rightarrow+\infty .
$$

This result yields as a confidence interval with asymptotic level $1-\alpha$ for the tail index $\gamma_{0}$, the set of all $\gamma$ values such that $l\left(\gamma, \hat{\sigma}_{\gamma}\right) \leq c_{\alpha}$, where $c_{\alpha}$ is the $1-\alpha$ quantile of the $\chi^{2}(1)$ distribution.

Remark 1. Note that the restriction $r<1 / 3$ could be reduced to $r<1 / 2$, but this would unnecessarily complicate the proof since most of the time $r$ should be chosen negative (see J. Zhang (2007) for a discussion). 


\section{Simulations}

\subsection{Simulations for the couple $(\gamma, \sigma)$}

In this subsection, we present a small simulation study in order to investigate the performance of our proposed method for constructing confidence regions for the couple $\left(\gamma_{0}, \sigma_{0 n}\right)$ based on empirical likelihood techniques (Theorem 1). We compare empirical coverage probabilities of the confidence regions (with nominal level 0.95) produced by our empirical likelihood method (EL(0.95)), the normal approximation for the Maximum Likelihood estimators $(\mathrm{ML}(0.95))$ and the normal approximation for the estimators proposed in J. Zhang (2007) (Zhang(0.95)).

Before giving more details on the simulation study, let us make the following remarks.

Remark 2. The CLT for the GPD parameters stated in J. Zhang (2007) has been proved when the underlying distribution is a pure GPD : using Theorem 2.1 in J. Zhang (2007) (which asserts the existence and unicity of the estimator), and following the same methodology that led to Proposition 2 in Section 4.1, the consistency of the sequence $\left(\hat{\gamma}, \hat{\sigma}_{n}\right)$ can be obtained (details are omitted here) and accordingly, by classical methods, the asymptotic normality follows in the general case of the underlying distribution belonging to the Fréchet maximum domain of attraction (assuming that $\sqrt{k_{n}} a_{n} \rightarrow 0$ ). We will therefore use the convergence in distribution of $\sqrt{k_{n}}\left(\hat{\gamma}-\gamma_{0}, \hat{\sigma}_{n} / \sigma_{0 n}-1\right)$ to $\mathcal{N}_{2}\left(0, \Sigma\left(\gamma_{0}, r\right)\right)$, where

$$
\Sigma(\gamma, r):=\left(\begin{array}{cc}
(1-r)\left(1+\left(2 \gamma^{2}+2 \gamma+r\right) /(1-2 r)\right. & -1-\left(r^{2}+\gamma^{2}+\gamma\right) /(1-2 r) \\
-1-\left(r^{2}+\gamma^{2}+\gamma\right) /(1-2 r) & 2+\left((r-\gamma)^{2}+2 \gamma\right) /(1-2 r)
\end{array}\right)
$$

and consider the corresponding Wald-type confidence region, based on approximating the distribution of the statistic $\left\|\sqrt{k_{n}}(\Sigma(\hat{\gamma}, r))^{-1 / 2}\left(\hat{\gamma}-\gamma_{0}, \hat{\sigma}_{n} / \sigma_{0 n}-1\right)\right\|^{2}$ by $\chi^{2}(2)$. The same methodology is applied for constructing confidence regions based on the Maximum Likelihood estimators (see R. L. Smith (1987)).

Remark 3. The tuning parameter $r$ in (4) is chosen equal to $-1 / 2$ (as suggested in J. Zhang (2007), without any prior information). The empirical likelihood confidence region is based on a Fisher calibration rather than a $\chi^{2}$ calibration, as suggested in A.B. Owen (1990) : concretely, this means that our confidence region consists in the set of all $(\gamma, \sigma)$ such that $l(\gamma, \sigma) \leq f$ where $\mathbb{P}\left(\left(2\left(k_{n}-1\right) /\left(k_{n}-2\right)\right) F\left(2, k_{n}-2\right) \leq f\right)=0.95$. Indeed, it has been empirically observed that it (uniformly in $k_{n}$ ) produces slightly better results in terms of coverage probability.

The simulations below are based on 2000 random samples of size $n=1000$, generated from the following two distributions: the Fréchet distribution with parameter $\gamma>0$ given by $F(x)=\exp \left(-x^{-1 / \gamma}\right)$ (the results for $\gamma=1$ and $1 / 4$ are presented) and the Burr distribution with positive parameters $\lambda$ and $\tau$ given by $F(x)=\left(1+x^{\tau}\right)^{-1 / \lambda}$ (the results for $(\lambda, \tau)=(1,1)$ and $(2,2)$ are presented). Note that the tail index for the Burr distribution is $\gamma=(\lambda \tau)^{-1}$.

Coverage probabilities EL(0.95), ML(0.95) and Zhang(0.95) are plotted against different values of $k_{n}$, the number of excesses used. Figure 1 seems to indicate that our method performs better in terms of coverage probability, and additional models not presented here almost always led to the same ordering : EL better than the bivariate CLT of 
Zhang's estimator, itself better than the CLT for the MLE. However, we have observed that the overall results are not very satisfactory when the tail index $\gamma$ is small.

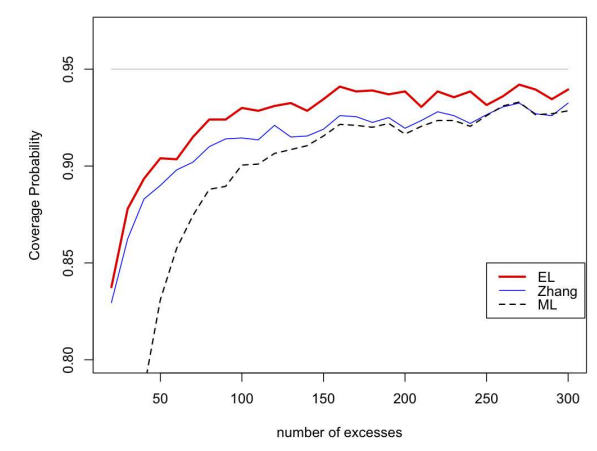

(a) Coverage Probability for Burr $(1,1)$ model, $n=1000$

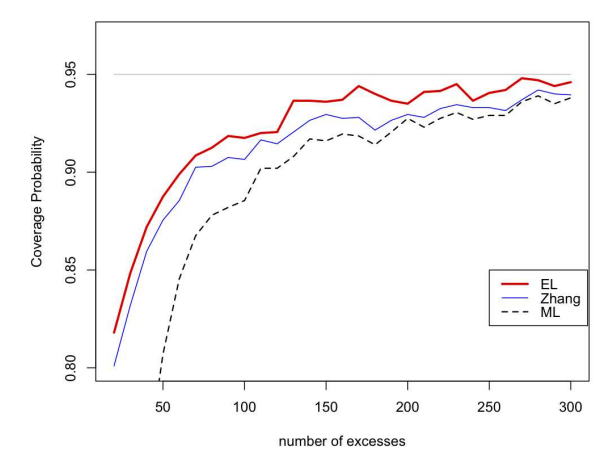

(c) Coverage Probability for Frechet(1) model, $n=1000$

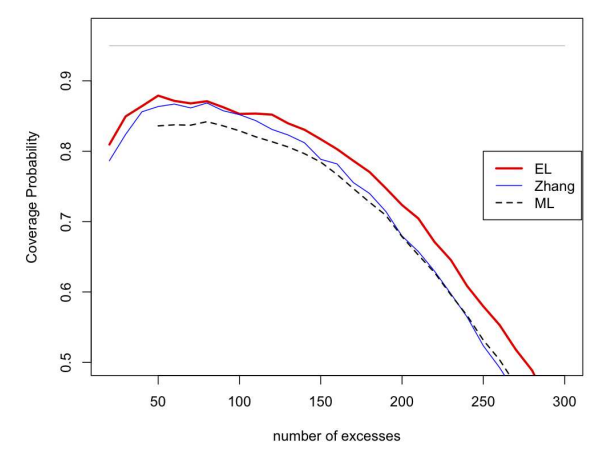

(b) Coverage Probability for $\operatorname{Burr}(2,2)$ model, $n=1000$

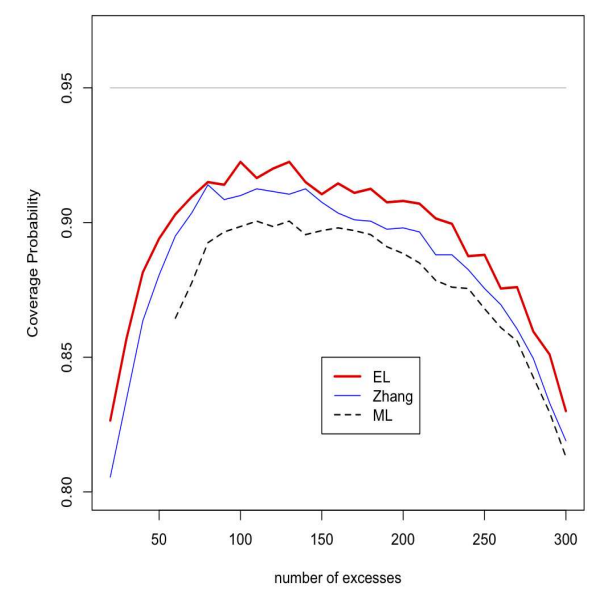

(d) Coverage Probability for Frechet $(1 / 4)$ model, $n=1000$

Figure 1: Coverage Probability for $\operatorname{Burr}(1,1), \operatorname{Burr}(2,2)$, Frechet(1), Frechet(1/4) as a function of the number of excesses $k_{n}$. The dashed line is for ML, the thin solid line for Zhang, and the thick solid line for EL.

One can wonder if the improvement in coverage probabilities is due to the fact that our confidence regions are wider than in the ML and Zhang cases. In practice, it seems in fact that the three confidence regions have comparable sizes (our confidence region being even a bit smaller). Figure 2 shows these three regions for a simulated $\operatorname{Burr}(1,1)$ random sample with $n=1000$ et $k_{n}=200$.

Remark 4. it should be noted that some computational problems occurred when trying 


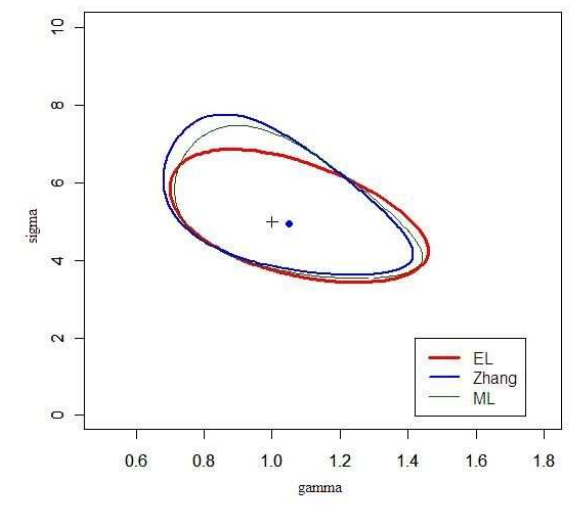

Figure 2: Confidence regions for a sample of $\operatorname{Burr}(1,1)$. The thinner line is for ML, the thicker one is for EL and the other for Zhang.

to calculate the maximum likelihood estimators. This explains why in some of the figures above, a piece of the curve is lacking for the smaller values of $k_{n}$ (the computation was performed by using the function fpot of the evd package of $\mathrm{R}$ ).

\subsection{Simulations for $\gamma$}

In this subsection, we present another small simulation study which is now concerned by the performance of our method for constructing confidence intervals for the tail index $\gamma$ based on profile empirical likelihood techniques (Theorem 2). We compare empirical coverage probabilities of the confidence intervals for our empirical profile likelihood method (ELW(0.95)), the empirical likelihood method proposed in J.C. Lu and L. Peng (2002) and based on the Hill estimator $(\operatorname{ELP}(0.95))$ and finally the normal approximation for the estimator of $\gamma$ proposed in J. Zhang (2007) (Zhang(0.95)). Note that for $\operatorname{ELP}(0.95)$, we used the exponential calibration in order to calculate the critical value, as prescribed in L. Peng and Y. Qi (2006). As before, the Fisher calibration was preferred to the $\chi^{2}$ one in order to compute the critical value in our case. We worked with the same two distributions as in the couple case : the results are presented for $\gamma=1$ and $1 / 4$ in the Fréchet case and $(\lambda, \tau)=(1,1)$ and $(2,2)$ in the Burr one.

Empirical coverage probabilities are plotted against different values of $k_{n}$. Contrary to the couple framework, Figure 3 shows no significant improvement with respect to Zhang's CLT based confidence intervals, which itself shows some problems of undercoverage. The EL-based confidence intervals of Lu, Peng and Qi, show quite satisfactory coverage probabilities, but on a range of values of $k_{n}$ which is sometimes very narrow (which is a common phenomenon in POT methodology) : this drawback is much less present for the confidence intervals based on Zhang's equations, which show a better stability against the choice of $k_{n}$. Moreover, simulations (not presented here) showed that the widths of the ELP interval and the ELW interval are comparable, and smaller than the width of the interval based on Zhang's CLT. 


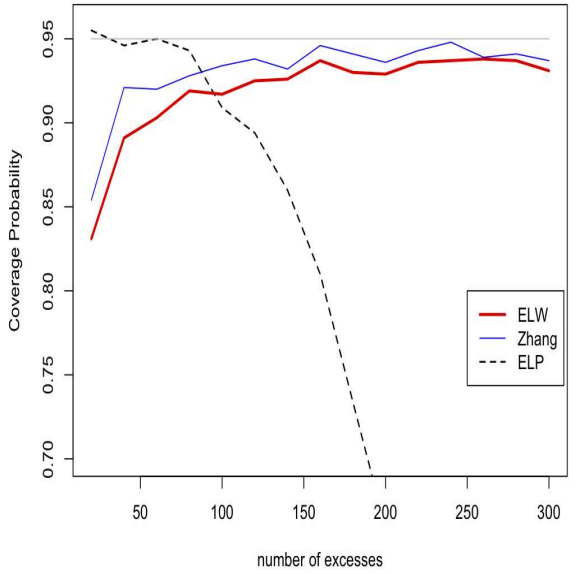

(a) Coverage Probability for Burr $(1,1)$ model, $n=1000$

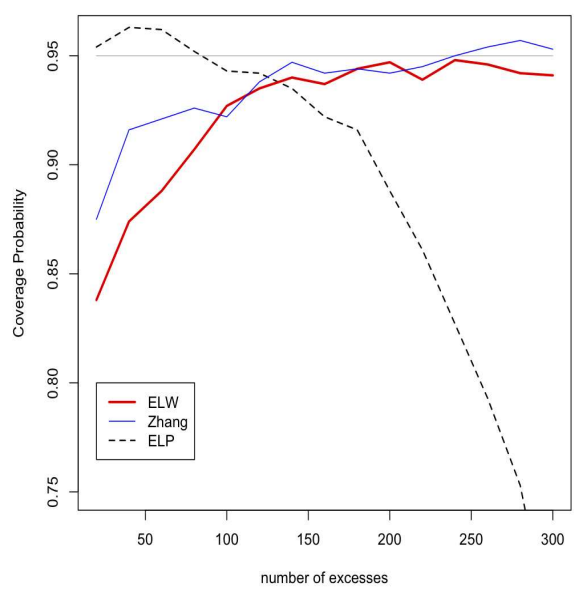

(c) Coverage Probability for Frechet(1) model, $n=1000$

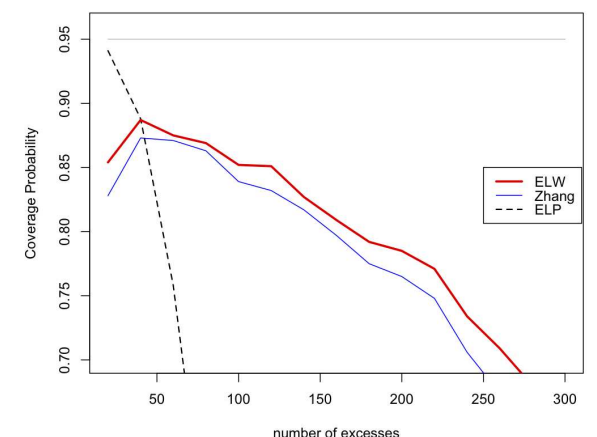

(b) Coverage Probability for $\operatorname{Burr}(2,2)$ model, $n=1000$

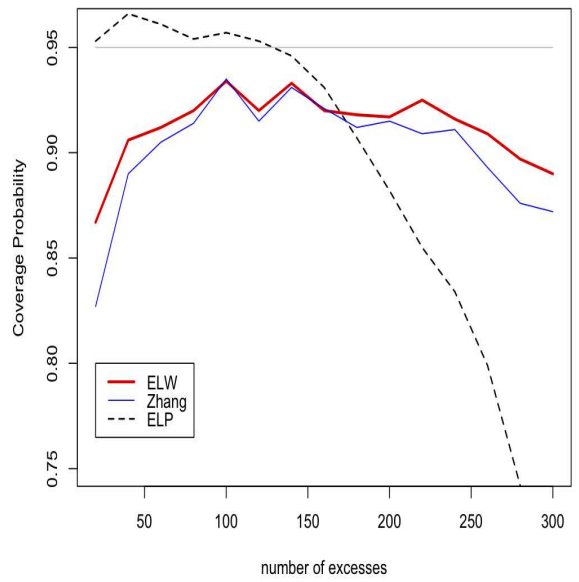

(d) Coverage Probability for Frechet(1/4) model, $n=1000$

Figure 3: Coverage Probability for $\operatorname{Burr}(1,1), \operatorname{Burr}(2,2)$, Frechet(1), Frechet(1/4) as a function of the number of excesses $k_{n}$. The dashed line is for $E L P$, the thin solid line for Zhang, and the thick solid line for $E L W$. 
Remark 5. the computation of the profile empirical likelihood $l\left(\gamma_{0}, \hat{\sigma}_{\gamma_{0}}\right)$ was performed using a classical descent algorithm, taking profit of some convexity properties of the profile empirical likelihood function. Computational details and files can be obtained from the authors (some of them are downloadable on the first author's webpage).

\section{Proofs}

Note that we will prove Theorem 2 before Theorem 1 because its proof is more involved and largely includes what is needed to prove Theorem 1.

\subsection{Proof of Theorem 2}

From now on we work conditionally on $\left\{N_{n}=k_{n}\right\}$ for some given sequence $\left(k_{n}\right)$ satisfying $\sqrt{k_{n}} a_{n} \rightarrow 0$ as $n \rightarrow \infty$.

Let $U_{1}, \ldots, U_{k_{n}}$ denote independent uniform random variables on $[0,1]$. Noticing that $\left(Y_{1}, \ldots, Y_{k_{n}}\right)$ has the same joint distribution as $\left(\tilde{Y}_{1}, \ldots, \tilde{Y}_{k_{n}}\right)$ defined by

$$
\tilde{Y}_{i}:=\bar{F}_{u_{n}}^{-1}\left(U_{i}\right)=U\left(1 /\left(U_{i} \bar{F}\left(u_{n}\right)\right)\right)-U\left(1 / \bar{F}\left(u_{n}\right)\right)
$$

we see that it suffices to prove Theorem 2 with $\tilde{Y}_{i}$ replacing $Y_{i}$ in the definition of the empirical likelihood. For simplicity we will write $Y_{i}$ instead of $\tilde{Y}_{i}$ in the sequel (note that these random variables are i.i.d. but their common distribution varies with $n$ ).

Now, defining $Z_{i, n}:=Y_{i} / \sigma_{0 n}, \lambda_{0}(\theta):=\lambda\left(\gamma_{0}, \sigma_{0 n} \theta\right)$ and $g_{0}(z, t):=g\left(z, \gamma_{0}, t\right)$, we see that $g_{0}\left(Z_{i, n}, \theta\right)=g\left(Y_{i}, \gamma_{0}, \sigma_{0 n} \theta\right)$, hence

$$
\begin{aligned}
l_{0}(\theta):=l\left(\gamma_{0}, \sigma_{0 n} \theta\right) & =2 \sum_{i=1}^{k_{n}} \log \left(1+<\lambda\left(\gamma_{0}, \sigma_{0 n} \theta\right), g\left(Y_{i}, \gamma_{0}, \sigma_{0 n} \theta\right)>\right) \\
& =2 \sum_{i=1}^{k_{n}} \log \left(1+<\lambda_{0}(\theta), g_{0}\left(Z_{i, n}, \theta\right)>\right) .
\end{aligned}
$$

With these preliminaries in mind, we thus need to prove that there exists some local minimizer $\hat{\theta}$ of $l_{0}(\cdot)$ in a neighborhood of $\theta_{0}=1$ such that $l_{0}(\hat{\theta}) \rightarrow \chi^{2}(1)$ in distribution, because $l_{0}(\hat{\theta})=l\left(\gamma_{0}, \hat{\sigma}_{\gamma_{0}}\right)$ with $\hat{\theta}=\hat{\sigma}_{\gamma_{0}} / \sigma_{0 n}$.

We now state in the following proposition some important results, which will be proved in Section 4.2 and will enable us to proceed with the proof of Theorem 2 following a plan very similar to that found in Y. S. Qin and J. Lawless (1994) (note that here the parameter is one-dimensional whereas the estimating function $g_{0}$ is $\mathbb{R}^{2}$-valued). We first introduce some important notations :

$$
\begin{aligned}
G_{n}(\theta) & :=\frac{1}{k_{n}} \sum_{i=1}^{k_{n}} g_{0}\left(Z_{i, n}, \theta\right) \\
B_{n}(\theta) & :=\frac{1}{k_{n}} \sum_{i=1}^{k_{n}} g_{0}\left(Z_{i, n}, \theta\right)\left(g_{0}\left(Z_{i, n}, \theta\right)\right)^{t} \\
A_{n}(\theta) & :=\frac{1}{k_{n}} \sum_{i=1}^{k_{n}} \frac{\partial g_{0}}{\partial \theta}\left(Z_{i, n}, \theta\right) \\
M_{n}(\theta) & :=\max _{i \leq k_{n}}\left\|g_{0}\left(Z_{i, n}, \theta\right)\right\|
\end{aligned}
$$

Note that, although the new parameter $\theta$ is scalar, we will write below $\|\theta\|$ instead of $|\theta|$ in order to emphasize the fact that the arguments described below can be applied to more general frameworks. The same is true about the fact that we use below the notation $\theta_{0}$ instead of simply the number 1 . 
Proposition 1. Suppose the assumptions of Theorem 2 are valid, and $Z$ is some random variable distributed as $G_{\gamma_{0}, 1}$. If $\mathcal{B}_{n}:=\left\{\theta \in \mathbb{R} ;\left\|\theta-\theta_{0}\right\| \leq k_{n}^{-1 / 3}\right\}$, then we have, conditionally to $\left\{N_{n}=k_{n}\right\}$ and as $n \rightarrow \infty$,

$$
\begin{aligned}
G_{n}\left(\theta_{0}\right) & =O\left(k_{n}^{-1 / 2}\left(\log \log k_{n}\right)^{1 / 2}\right) \text { a.s. } \\
\sqrt{k_{n}} G_{n}\left(\theta_{0}\right) & \stackrel{d}{\longrightarrow} \mathcal{N}(0, B) \\
M_{n}\left(\theta_{0}\right) & =o\left(\sqrt{k_{n}}\right) \text { a.s. } \\
M_{n}(\theta) & =o\left(k_{n}^{1 / 3}\right) \text { a.s. uniformly on } \mathcal{B}_{n} \\
A_{n}(\theta) & =A+o(1) \text { a.s. uniformly on } \mathcal{B}_{n}, \\
B_{n}(\theta) & =B+o(1) \text { a.s. uniformly on } \mathcal{B}_{n} \\
G_{n}(\theta) & =G_{n}\left(\theta_{0}\right)+(A+o(1))\left(\theta-\theta_{0}\right)=O\left(k_{n}^{-1 / 3}\right) \text { a.s. unif. on } \mathcal{B}_{n}
\end{aligned}
$$

where

$$
B:=\mathbb{E}\left[g_{0}\left(Z, \theta_{0}\right)\left(g_{0}\left(Z, \theta_{0}\right)\right)^{t}\right]=\left(\begin{array}{cc}
\gamma^{2} & \frac{\gamma r}{(1-r)^{2}} \\
\frac{\gamma r}{(1-r)^{2}} & \frac{r^{2}}{(1-2 r)(1-r)^{2}}
\end{array}\right)
$$

and

$$
A:=\mathbb{E}\left[\frac{\partial g_{0}}{\partial \theta}\left(Z, \theta_{0}\right)\right]=\left(\begin{array}{c}
-\frac{\gamma}{\gamma+1} \\
-\frac{r}{(1-r+\gamma)(1-r)}
\end{array}\right)
$$

Recall that $\lambda_{0}(\theta)$ is defined as the solution of the equation

$$
\frac{1}{k_{n}} \sum_{i=1}^{k_{n}}\left(1+<\lambda, g_{0}\left(Z_{i, n}, \theta\right)>\right)^{-1} g_{0}\left(Z_{i, n}, \theta\right)=0 .
$$

Therefore, for $\theta \in \mathcal{B}_{n}$, if $u=\lambda_{0}(\theta) /\left\|\lambda_{0}(\theta)\right\|$, usual calculations (see A.B. Owen (1990) for instance) lead to

$$
\left\|\lambda_{0}(\theta)\right\|\left(u^{t} B_{n}(\theta) u-M_{n}(\theta)\left\|G_{n}(\theta)\right\|\right) \leq\left\|G_{n}(\theta)\right\|
$$

for any $n$. Statements (11), (12), (15) and (13), (15), (16) thus respectively yield

$$
\left\|\lambda_{0}\left(\theta_{0}\right)\right\|=O\left(k_{n}^{-1 / 2}\right) \text { a.s. and }\left\|\lambda_{0}(\theta)\right\|=O\left(k_{n}^{-1 / 3}\right) \text { a.s. uniformly on } \mathcal{B}_{n} .
$$

Consequently, if we note $\gamma_{i, n}(\theta):=<\lambda_{0}(\theta), g_{0}\left(Z_{i, n}, \theta\right)>$, we have

$$
\max _{i \leq n}\left|\gamma_{i, n}(\theta)\right| \leq\left\|\lambda_{0}(\theta)\right\| M_{n}(\theta)=o(1) \text { a.s. and uniformly on } \mathcal{B}_{n}
$$

and, using $(1+x)^{-1}=1-x+x^{2}(1+x)^{-1}$ and the identity $0=k_{n}^{-1} \sum_{i=1}^{k_{n}}(1+$ $\left.\gamma_{i, n}(\theta)\right)^{-1} g_{0}\left(Z_{i, n}, \theta\right)$, we readily have

$$
\lambda_{0}(\theta)=\left(B_{n}(\theta)\right)^{-1} G_{n}(\theta)+\left(B_{n}(\theta)\right)^{-1} R_{n}(\theta)
$$

where $R_{n}(\theta)=k_{n}^{-1} \sum_{i=1}^{k_{n}}\left(1+\gamma_{i, n}(\theta)\right)^{-1}\left(\gamma_{i, n}(\theta)\right)^{2} g_{0}\left(Z_{i, n}, \theta\right)$. Since for $n$ sufficiently large we have $\left\|R_{n}(\theta)\right\| \leq 2 k_{n}^{-1} \sum_{i=1}^{k_{n}}\left\|\lambda_{0}(\theta)\right\|^{2}\left\|g_{0}\left(Z_{i, n}, \theta\right)\right\|^{3} \leq 2\left\|\lambda_{0}(\theta)\right\|^{2} M_{n}(\theta) \operatorname{tr}\left(B_{n}(\theta)\right)$, relations (12), (13), (15) and (18) imply the following crucial relations (the second one holding uniformly in $\left.\theta \in \mathcal{B}_{n}\right)$

$\lambda_{0}\left(\theta_{0}\right)=\left(B_{n}\left(\theta_{0}\right)\right)^{-1} G_{n}\left(\theta_{0}\right)+o\left(k_{n}^{-1 / 2}\right) \quad$ a.s. and $\quad \lambda_{0}(\theta)=\left(B_{n}(\theta)\right)^{-1} G_{n}(\theta)+o\left(k_{n}^{-1 / 3}\right)$ a.s.. 
Using the Taylor expansion $\log (1+x)=x-\frac{1}{2} x^{2}+\frac{1}{3} x^{3}(1+\xi)^{-3}$ (for some $\xi$ between 0 and $x$ ) and statement (19), we can proceed as above and obtain

$$
l_{0}(\theta)=2 \sum_{i=1}^{k_{n}} \log \left(1+\gamma_{i, n}(\theta)\right)=2 \sum_{i=1}^{k_{n}} \gamma_{i, n}(\theta)-\sum_{i=1}^{k_{n}}\left(\gamma_{i, n}(\theta)\right)^{2}+R_{n}^{\prime}(\theta)
$$

where, for $n$ sufficiently large, $\left\|R_{n}^{\prime}(\theta)\right\| \leq \frac{16}{3} \sum_{i=1}^{k_{n}}\left|\gamma_{i, n}(\theta)\right|^{3}=o(1) \sum_{i=1}^{k_{n}}\left(\gamma_{i, n}(\theta)\right)^{2}$. Using relation (20) as well as (15) and (16), we have for $\left\|\theta-\theta_{0}\right\| \leq k_{n}^{-1 / 3}$,

$$
\begin{aligned}
\sum_{i=1}^{k_{n}} \gamma_{i, n}(\theta)=k_{n} \lambda_{0}(\theta)^{t} G_{n}(\theta) & =k_{n}\left(G_{n}(\theta)+o\left(k_{n}^{-1 / 3}\right)\right)^{t}\left(B_{n}(\theta)\right)^{-1} G_{n}(\theta) \\
& =k_{n}\left(G_{n}(\theta)\right)^{t} B^{-1} G_{n}(\theta)+o\left(k_{n}^{1 / 3}\right)
\end{aligned}
$$

and similarly, $\sum_{i=1}^{k_{n}}\left(\gamma_{i, n}(\theta)\right)^{2}=k_{n}\left(G_{n}(\theta)\right)^{t} B^{-1} G_{n}(\theta)+o\left(k_{n}^{1 / 3}\right)$. Therefore, if $\theta=$ $\theta_{0}+u k_{n}^{-1 / 3}$ with $\|u\|=1$, using (16) and the almost sure bound (10) for $G_{n}\left(\theta_{0}\right)$, we obtain

$$
\begin{aligned}
l_{0}(\theta) & =k_{n}\left(G_{n}(\theta)\right)^{t} B^{-1} G_{n}(\theta)+o\left(k_{n}^{1 / 3}\right) \\
& =k_{n}^{1 / 3}\left[k_{n}^{1 / 3} G_{n}\left(\theta_{0}\right)+(A+o(1)) u\right]^{t} B^{-1}\left[k_{n}^{1 / 3} G_{n}\left(\theta_{0}\right)+(A+o(1)) u\right]+o\left(k_{n}^{1 / 3}\right) \\
& =k_{n}^{1 / 3} u^{t} A^{t} B^{-1} A u+o\left(k_{n}^{1 / 3}\right) .
\end{aligned}
$$

Consequently, if $a>0$ denotes the smallest eigenvalue of $A^{t} B^{-1} A$ and $\left.\epsilon \in\right] 0, a[$, we have for $n$ sufficiently large

$$
l_{0}(\theta) \geq(a-\epsilon) k_{n}^{1 / 3} \text { almost surely and uniformly for } \theta \in \operatorname{cl}\left(\mathcal{B}_{n}\right) .
$$

On the other hand, we obtain in a similar manner

$$
l_{0}\left(\theta_{0}\right)=\left(\sqrt{k_{n}} G_{n}\left(\theta_{0}\right)\right)^{t} B^{-1}\left(\sqrt{k_{n}} G_{n}\left(\theta_{0}\right)\right)+o(1) \quad \text { (a.s.) }
$$

which converges in distribution to $\chi^{2}(2)$ in view of (11), and is also $o\left(\log \log k_{n}\right)$ almost surely, thanks to (10).

We have thus proved the following

Proposition 2. Under the conditions of Theorem 2, and as $n \rightarrow \infty$ with probability one, the empirical log-likelihood ratio $l_{0}(\cdot)$ admits a local minimizer $\hat{\theta}$ in the interior of the ball $\mathcal{B}_{n}=\left\{\theta \in \mathbb{R} ;\left\|\theta-\theta_{0}\right\| \leq k_{n}^{-1 / 3}\right\}$. This means that almost surely, for $n$ large, there exists a local minimizer $\hat{\sigma}_{\gamma_{0}}$ of the profile empirical log-likelihood $\sigma \mapsto l\left(\gamma_{0}, \sigma\right)$ such that $\hat{\sigma}_{\gamma_{0}} / \sigma_{0 n}$ is close to 1 with rate $k_{n}^{-1 / 3}$.

Now that we have identified some empirical likelihood estimator $\hat{\theta}$ and proved it consistently estimates $\theta_{0}$, we want to identify its asymptotic distribution, which will enable us to obtain the convergence in distribution of $l_{0}(\hat{\theta})$ towards $\chi^{2}(1)$.

As it is done in Y. S. Qin and J. Lawless (1994), we introduce the functions defined on 
$\mathbb{R} \times \mathbb{R}^{2}$

$$
\begin{aligned}
Q_{1, n}(\theta, \lambda) & =\frac{1}{k_{n}} \sum_{i=1}^{k_{n}}\left(1+<\lambda, g_{0}\left(Z_{i, n}, \theta\right)>\right)^{-1} g_{0}\left(Z_{i, n}, \theta\right) \\
Q_{2, n}(\theta, \lambda) & =\frac{1}{k_{n}} \sum_{i=1}^{k_{n}}\left(1+<\lambda, g_{0}\left(Z_{i, n}, \theta\right)>\right)^{-1}\left(\frac{\partial g_{0}}{\partial \theta}\left(Z_{i, n}, \theta\right)\right)^{t} \lambda
\end{aligned}
$$

and see that $(\forall \theta) Q_{1, n}\left(\theta, \lambda_{0}(\theta)\right)=0$ (by definition of $\lambda_{0}(\theta)$ ), $Q_{1, n}(\theta, 0)=G_{n}(\theta)$, and $Q_{2, n}\left(\theta, \lambda_{0}(\theta)\right)=\left(\partial l_{0} / \partial \theta\right)(\theta)$, which is null at $\theta=\hat{\theta}$.

A Taylor expansion of $Q_{1, n}$ and $Q_{2, n}$ between $\left(\theta_{0}, 0\right)$ and $\left(\hat{\theta}, \lambda_{0}(\hat{\theta})\right)$ shows that there exists some $\left(\theta_{n}^{*}, \lambda_{n}^{*}\right)$ satisfying $\left\|\theta_{n}^{*}-\theta_{0}\right\| \leq\left\|\hat{\theta}-\theta_{0}\right\| \leq k_{n}^{-1 / 3},\left\|\lambda_{n}^{*}\right\| \leq\left\|\lambda_{0}(\hat{\theta})\right\|=O\left(k_{n}^{-1 / 3}\right)$ (thanks to Proposition 2 and (18)), and such that

$$
\left(\begin{array}{l}
0 \\
0
\end{array}\right)=\left(\begin{array}{c}
Q_{1, n}\left(\hat{\theta}, \lambda_{0}(\hat{\theta})\right) \\
Q_{2, n}\left(\hat{\theta}, \lambda_{0}(\hat{\theta})\right)
\end{array}\right)=\left(\begin{array}{c}
Q_{1, n}\left(\theta_{0}, 0\right) \\
Q_{2, n}\left(\theta_{0}, 0\right)
\end{array}\right)-S_{n}\left(\theta_{n}^{*}, \lambda_{n}^{*}\right)\left(\begin{array}{c}
\hat{\theta}-\theta_{0} \\
\lambda_{0}(\hat{\theta})
\end{array}\right)
$$

where

$$
S_{n}(\theta, \lambda):=\left.\left(\begin{array}{ll}
-\partial Q_{1, n} / \partial \theta & -\partial Q_{1, n} / \partial \lambda \\
-\partial Q_{2, n} / \partial \theta & -\partial Q_{2, n} / \partial \lambda
\end{array}\right)\right|_{(\theta, \lambda)}
$$

Differential calculus leads to

$$
S_{n}\left(\theta_{0}, 0\right)=\left(\begin{array}{cc}
-\frac{1}{k_{n}} \sum_{i=1}^{k_{n}} \frac{\partial g}{\partial \theta}\left(Z_{i, n}, \theta_{0}\right) & \frac{1}{k_{n}} \sum_{i=1}^{k_{n}} g\left(Z_{i, n}, \theta_{0}\right)\left(g\left(Z_{i, n}, \theta_{0}\right)\right)^{t} \\
0 & -\frac{1}{k_{n}} \sum_{i=1}^{k_{n}}\left(\frac{\partial g}{\partial \theta}\left(Z_{i, n}, \theta_{0}\right)\right)^{t}
\end{array}\right)
$$

thus, defining $V:=\left(A^{t} B^{-1} A\right)^{-1}$, relations (15) and (14) imply that $S_{n}\left(\theta_{0}, 0\right)$ converges to the matrix

$$
S=\left(\begin{array}{cc}
-A & B \\
0 & -A^{t}
\end{array}\right)
$$

which is invertible with

$$
S^{-1}=\left(\begin{array}{cc}
C & D \\
E & F
\end{array}\right):=\left(\begin{array}{cc}
-V A^{t} B^{-1} & -V \\
B^{-1}\left(I-A V A^{t} B^{-1}\right) & -B^{-1} A V
\end{array}\right)
$$

After tedious calculations and use of many of the statements previously derived from Proposition 1 , it can be proved that $\left\|S_{n}\left(\theta_{n}^{*}, \lambda_{n}^{*}\right)-S_{n}\left(\theta_{0}, 0\right)\right\|=o_{\mathbb{P}}(1)$ as $n \rightarrow \infty$. Consequently, we obtain, for $n$ sufficiently large

$$
\left(\begin{array}{c}
\sqrt{k_{n}}\left(\hat{\theta}-\theta_{0}\right) \\
\sqrt{k_{n}} \lambda_{0}(\hat{\theta})
\end{array}\right)=S^{-1}\left(\begin{array}{c}
\sqrt{k_{n}} G_{n}\left(\theta_{0}\right)+o_{\mathbb{P}}\left(\sqrt{k_{n}} \delta_{n}\right) \\
o_{\mathbb{P}}\left(\sqrt{k_{n}} \delta_{n}\right)
\end{array}\right) \quad \text { where } \quad \delta_{n}:=\left\|\hat{\theta}-\theta_{0}\right\|+\left\|\lambda_{0}(\hat{\theta})\right\|
$$

We already know that $\delta_{n}=O\left(k_{n}^{-1 / 3}\right)$, but now (21) implies that $\delta_{n}=O\left(k_{n}^{-1 / 2}\right)$ and therefore we have proved that

$$
\begin{aligned}
\sqrt{k_{n}}\left(\hat{\theta}-\theta_{0}\right) & =C \sqrt{k_{n}} G_{n}\left(\theta_{0}\right)+o_{\mathbb{P}}(1) \quad \stackrel{d}{\rightarrow} \mathcal{N}\left(0, C B C^{t}\right)=\mathcal{N}\left(0,\left(A^{t} B^{-1} A\right)^{-1}\right) \\
\sqrt{k_{n}} \lambda_{0}(\hat{\theta}) & =E \sqrt{k_{n}} G_{n}\left(\theta_{0}\right)+o_{\mathbb{P}}(1) \quad \stackrel{d}{\rightarrow} \mathcal{N}\left(0, E B E^{t}\right)=\mathcal{N}(0, E)
\end{aligned}
$$

where we have used the fact that the matrix $E$ is symmetric and such that $E B E=$ $E$, because $I-A V A^{t} B^{-1}$ is idempotent (note that the rank of $E$ is 2 minus that of 
$A V A^{t} B^{-1}$, i.e. $\left.\operatorname{rank}(E)=1\right)$.

Applying relation (16) to $\theta=\hat{\theta}$, relation (22) yields

$$
\begin{aligned}
\sqrt{k_{n}} G_{n}(\hat{\theta})=\sqrt{k_{n}} G_{n}\left(\theta_{0}\right)+A \sqrt{k_{n}}\left(\hat{\theta}-\theta_{0}\right)+o_{\mathbb{P}}(1)=(I+A C) \sqrt{k_{n}} G_{n}\left(\theta_{0}\right)+o_{\mathbb{P}}(1) \\
=B E\left(\sqrt{k_{n}} G_{n}\left(\theta_{0}\right)+o_{\mathbb{P}}(1)\right)
\end{aligned}
$$

and this leads to the following appropriate development for $l_{0}(\hat{\theta})$, using $(23)$ and (15):

$$
\begin{aligned}
l_{0}(\hat{\theta}) & =2 k_{n}\left(\lambda_{0}(\hat{\theta})\right)^{t} G_{n}(\hat{\theta})-k_{n}\left(\lambda_{0}(\hat{\theta})\right)^{t} B_{n}(\hat{\theta}) \lambda_{0}(\hat{\theta})+R_{n}^{\prime}(\hat{\theta}) \\
& =\left(\sqrt{k_{n}} G_{n}\left(\theta_{0}\right)+o_{\mathbb{P}}(1)\right)^{t}(E B E)\left(\sqrt{k_{n}} G_{n}\left(\theta_{0}\right)+o_{\mathbb{P}}(1)\right)+o_{\mathbb{P}}(1)+R_{n}^{\prime}(\hat{\theta}) \\
& =\left(\sqrt{k_{n}} G_{n}\left(\theta_{0}\right)\right)^{t} E\left(\sqrt{k_{n}} G_{n}\left(\theta_{0}\right)\right)+o_{\mathbb{P}}(1)+R_{n}^{\prime}(\hat{\theta})
\end{aligned}
$$

where $\left|R_{n}^{\prime}(\hat{\theta})\right| \leq o_{\mathbb{P}}(1) \sum_{i=1}^{k_{n}}\left(\gamma_{i, n}(\hat{\theta})\right)^{2}=o_{\mathbb{P}}(1)\left(\left(\sqrt{k_{n}} G_{n}\left(\theta_{0}\right)\right)^{t} E\left(\sqrt{k_{n}} G_{n}\left(\theta_{0}\right)\right)+o_{\mathbb{P}}(1)\right)$.

According to proposition (viii) p.524 of S.S. Rao (1984), since $\sqrt{k_{n}} G_{n}\left(\theta_{0}\right)$ converges in distribution to $\mathcal{N}(0, B)$, and $E B E=E$ with $\operatorname{rank}(E B)=1$, the quadratic form $\left(\sqrt{k_{n}} G_{n}\left(\theta_{0}\right)\right)^{t} E\left(\sqrt{k_{n}} G_{n}\left(\theta_{0}\right)\right)$ converges in distribution to $\chi^{2}(2-1)=\chi^{2}(1)$, and Theorem 2 is proved.

\subsection{Proof of Proposition 1}

Note that throughout the whole proof, we will write $\gamma$ instead of $\gamma_{0}$ for convenience.

\subsubsection{Proof of (10) and (11)}

Let us define

$$
Z_{i}=\frac{U_{i}^{-\gamma}-1}{\gamma} \text { and } \Delta_{i}(\theta)=g_{0}\left(Z_{i, n}, \theta\right)-g_{0}\left(Z_{i}, \theta\right)
$$

so that $\left(Z_{i}\right)_{1 \leq i \leq k_{n}}$ is an i.i.d. sequence with common distribution $\operatorname{GPD}(\gamma, 1)$ and

$$
G_{n}\left(\theta_{0}\right)=\frac{1}{k_{n}} \sum_{i=1}^{k_{n}} g_{0}\left(Z_{i}, \theta_{0}\right)+\frac{1}{k_{n}} \sum_{i=1}^{k_{n}} \Delta_{i}\left(\theta_{0}\right) .
$$

If $r<\frac{1}{2}, B$ is well defined as the covariance matrix of $g_{0}\left(Z_{1}, \theta_{0}\right)$ (a straightforward calculation gives the expression of $B$ ), and consequently the LIL and CLT imply that

$\frac{1}{k_{n}} \sum_{i=1}^{k_{n}} g_{0}\left(Z_{i}, \theta_{0}\right)=O\left(k_{n}^{-1 / 2}\left(\log \log k_{n}\right)^{1 / 2}\right)$ a.s. and $\frac{1}{\sqrt{k_{n}}} \sum_{i=1}^{k_{n}} g_{0}\left(Z_{i}, \theta_{0}\right) \stackrel{d}{\longrightarrow} \mathcal{N}(0, B)$.

Therefore, according to (24) and to the assumption $\sqrt{k_{n}} a_{n} \rightarrow 0($ as $n \rightarrow+\infty)$, in order to prove (10) and (11) it remains to establish that

$$
\frac{1}{k_{n}} \sum_{i=1}^{k_{n}} \Delta_{i}\left(\theta_{0}\right)=O\left(a_{n}\right) \text { a.s. }
$$

Since we can take $\sigma_{0 n}:=\sigma_{0}\left(u_{n}\right)=\frac{1}{F\left(u_{n}\right)} U^{\prime}\left(1 / \bar{F}\left(u_{n}\right)\right)$, and recalling that we consider $Y_{i}=U\left(1 /\left(U_{i} \bar{F}\left(u_{n}\right)\right)\right)-U\left(1 / \bar{F}\left(u_{n}\right)\right)$, the application of the Potter-type bounds (8) to 
$t=1 / \bar{F}\left(u_{n}\right)$ and $x=1 / U_{i}$ yields, for all $\epsilon>0$ and $n$ sufficiently large,

$$
(1-\epsilon) U_{i}^{\epsilon} K_{\gamma, \rho}\left(1 / U_{i}\right) \leq \frac{Z_{i, n}-Z_{i}}{a_{n}} \leq(1+\epsilon) U_{i}^{-\epsilon} K_{\gamma, \rho}\left(1 / U_{i}\right) \text { a.s. }
$$

In the sequel, we will consider $a_{n}>0$ for large $n$ (the case $a_{n}<0$ being similar) and note $K_{i}=K_{\gamma, \rho}\left(1 / U_{i}\right)$, as well as $\Delta_{i}^{1}(\theta)$ and $\Delta_{i}^{2}(\theta)$ the two components of $\Delta_{i}(\theta)$.

(i) Control of $\Delta_{i}^{1}\left(\theta_{0}\right)$

$$
\Delta_{i}^{1}\left(\theta_{0}\right)=\ln \left(1+\gamma Z_{i, n}\right)-\ln \left(1+\gamma Z_{i}\right)=\ln \left(1+\gamma U_{i}^{\gamma}\left(Z_{i, n}-Z_{i}\right)\right) .
$$

Use of (26) leads to the following bounds (for all $\epsilon>0$ and $n$ sufficiently large),

$$
\frac{1}{a_{n}} \ln \left(1+a_{n} \gamma(1-\epsilon) U_{i}^{\gamma+\epsilon} K_{i}\right) \leq \frac{\Delta_{i}^{1}\left(\theta_{0}\right)}{a_{n}} \leq \frac{1}{a_{n}} \ln \left(1+a_{n} \gamma(1+\epsilon) U_{i}^{\gamma-\epsilon} K_{i}\right) \quad \text { a.s. }
$$

If we set $B_{i}^{+}:=\gamma(1+\epsilon) U_{i}^{\gamma-\epsilon} K_{i}$ and $B_{i}^{-}:=\gamma(1-\epsilon) U_{i}^{\gamma+\epsilon} K_{i}$, Lemmas 3 and 5 (stated and proved in the Appendix) imply that $B_{i}^{+}$and $B_{i}^{-}$are both square integrable and therefore $\max _{i \leq k_{n}} a_{n} B_{i}^{+}$and $\max _{i \leq k_{n}} a_{n} B_{i}^{-}$are both, almost surely, $o\left(\sqrt{k_{n}} a_{n}\right)$, which is $o(1)$ according to our assumption on $\left(k_{n}\right)$.

Consequently, the inequality $\frac{2}{3} x \leq \ln (1+x) \leq x(\forall x \in[0,1 / 2])$ yields the following bounds, for all $\epsilon>0$ and $n$ sufficiently large,

$$
\frac{2}{3} \frac{1}{k_{n}} \sum_{i=1}^{k_{n}} B_{i}^{-} \leq \frac{1}{k_{n}} \sum_{i=1}^{k_{n}} \frac{\Delta_{i}^{1}\left(\theta_{0}\right)}{a_{n}} \leq \frac{1}{k_{n}} \sum_{i=1}^{k_{n}} B_{i}^{+} \text {a.s. }
$$

and therefore, for every $\epsilon>0$,

$$
\begin{aligned}
\frac{2}{3} \gamma(1-\epsilon) \mathbb{E}\left(U_{1}^{\gamma+\epsilon} K_{1}\right) & \leq \liminf \frac{1}{k_{n}} \sum_{i=1}^{k_{n}} \frac{\Delta_{i}^{1}\left(\theta_{0}\right)}{a_{n}} \\
& \leq \lim \sup \frac{1}{k_{n}} \sum_{i=1}^{k_{n}} \frac{\Delta_{i}^{1}\left(\theta_{0}\right)}{a_{n}} \leq \gamma(1+\epsilon) \mathbb{E}\left(U_{1}^{\gamma-\epsilon} K_{1}\right) .
\end{aligned}
$$

Letting $\epsilon$ go to 0 gives $a_{n}^{-1} k_{n}^{-1} \sum_{i=1}^{k_{n}} \Delta_{i}^{1}\left(\theta_{0}\right)=O(1)$ a.s.

(ii) Control of $\Delta_{i}^{2}\left(\theta_{0}\right)$

$$
\Delta_{i}^{2}\left(\theta_{0}\right)=\left(1+\gamma Z_{i, n}\right)^{r / \gamma}-\left(1+\gamma Z_{i}\right)^{r / \gamma}=U_{i}^{-r}\left(\left(1+\gamma U_{i}^{\gamma}\left(Z_{i, n}-Z_{i}\right)\right)^{r / \gamma}-1\right) .
$$

In the case $r<0$ (the case $r>0$ is similar), use of (26) yields the following bounds for all $\epsilon>0$ and $n$ large

$$
\begin{aligned}
& \frac{U_{i}^{-r}}{a_{n}}\left(\left(1+(1+\epsilon) \gamma a_{n} U_{i}^{\gamma-\epsilon} K_{i}\right)^{r / \gamma}-1\right) \\
& \leq \frac{\Delta_{i}^{2}\left(\theta_{0}\right)}{a_{n}} \leq \frac{U_{i}^{-r}}{a_{n}}\left(\left(1+(1-\epsilon) \gamma a_{n} U_{i}^{\gamma+\epsilon} K_{i}\right)^{r / \gamma}-1\right)
\end{aligned}
$$

The inequality $\alpha x \leq(1+x)^{\alpha}-1 \leq \alpha c x\left(\forall x \in[0,1 / 2]\right.$, where $c=\left(\frac{3}{2}\right)^{\alpha-1}>0$ and $\alpha=r / \gamma<0)$ yields, for all $\epsilon>0$ and $n$ sufficiently large,

$$
r(1-\epsilon) \frac{1}{k_{n}} \sum_{i=1}^{k_{n}} U_{i}^{\gamma-r+\epsilon} K_{i} \leq \frac{1}{k_{n}} \sum_{i=1}^{k_{n}} \frac{\Delta_{i}^{2}\left(\theta_{0}\right)}{a_{n}} \leq r c(1+\epsilon) \frac{1}{k_{n}} \sum_{i=1}^{k_{n}} U_{i}^{\gamma-r-\epsilon} K_{i} \text { a.s. }
$$


Once again, Lemma 3 ensures that $\mathbb{E}\left(U_{i}^{\gamma-r \pm \epsilon} K_{i}\right)$ and $\mathbb{E}\left(\left(U_{i}^{\gamma-r \pm \epsilon} K_{i}\right)^{2}\right)$ are finite (because $r<1 / 2$ ), hence for sufficiently small $\epsilon>0$

$$
\begin{aligned}
r(1-\epsilon) \mathbb{E}\left(U_{1}^{\gamma-r+\epsilon} K_{1}\right) & \leq \liminf \frac{1}{k_{n}} \sum_{i=1}^{k_{n}} \frac{\Delta_{i}^{2}}{a_{n}} \\
& \leq \limsup \frac{1}{k_{n}} \sum_{i=1}^{k_{n}} \frac{\Delta_{i}^{2}\left(\theta_{0}\right)}{a_{n}} \leq r c(1+\epsilon) \mathbb{E}\left(U_{1}^{\gamma-r-\epsilon} K_{1}\right) \quad \text { a.s. }
\end{aligned}
$$

Letting $\epsilon$ go to 0 yields $a_{n}^{-1} k_{n}^{-1} \sum_{i=1}^{k_{n}} \Delta_{i}^{2}\left(\theta_{0}\right)=O(1)$ a.s. and therefore (25) is proved.

\subsubsection{Proof of (12) and (13)}

With $\Delta_{i}(\theta)$ and $Z_{i}$ being defined as previously, we have

$$
M_{n}(\theta)=\max _{i \leq k_{n}}\left\|g_{0}\left(Z_{i, n}, \theta\right)\right\| \leq \max _{i \leq k_{n}}\left\|g_{0}\left(Z_{i}, \theta\right)\right\|+\max _{i \leq k_{n}}\left\|\Delta_{i}(\theta)\right\| .
$$

Since the variables $g_{0}\left(Z_{i}, \theta_{0}\right)$ are square integrable, it comes (Lemma 5)

$$
\max _{i \leq k_{n}}\left\|g_{0}\left(Z_{i}, \theta_{0}\right)\right\|=o\left(\sqrt{k_{n}}\right) \text { a.s. }
$$

On the other hand, part 1 of Lemma 2 implies that for $\theta$ in $\mathcal{B}_{n},\left\|g_{0}(z, \theta)\right\|^{3} \leq G_{1}(z)$, for every $z \geq 0$ and $n$ sufficiently large. Since the variables $G_{1}\left(Z_{i}\right)$ are i.i.d. and integrable (part 4 of Lemma 2), using Lemma 5 we thus have

$$
\max _{i \leq k_{n}}\left\|g_{0}\left(Z_{i}, \theta\right)\right\|=o\left(k_{n}^{1 / 3}\right) \text { a.s. }
$$

We can now conclude the proof of (12) and (13) by showing that, uniformly for $\theta$ in $\mathcal{B}_{n}$, $\max _{i \leq k_{n}}\left|\Delta_{i}^{j}(\theta)\right|$ tends to 0 almost surely for $j=1$ or 2 . Reminding that $\gamma Z_{i}=U_{i}^{-\gamma}-1$, we can show that

$\Delta_{i}^{1}(\theta)=\ln \left(1+\gamma \frac{Z_{i, n}}{\theta}\right)-\ln \left(1+\gamma \frac{Z_{i}}{\theta}\right)=\ln \left\{1+\gamma U_{i}^{\gamma}\left(1+(\theta-1) U_{i}^{\gamma}\right)^{-1}\left(Z_{i, n}-Z_{i}\right)\right\}$.

Let $\delta>0$ and $\theta \in] 1-\delta, 1+\delta$. Using (26), we have the following bounds (for all $\epsilon>0$ and $n$ sufficiently large),

$$
\frac{1}{a_{n}} \ln \left(1+a_{n} \gamma\left(\frac{1-\epsilon}{1+\delta}\right) U_{i}^{\gamma+\epsilon} K_{i}\right) \leq \frac{\Delta_{i}^{1}(\theta)}{a_{n}} \leq \frac{1}{a_{n}} \ln \left(1+a_{n} \gamma\left(\frac{1+\epsilon}{1-\delta}\right) U_{i}^{\gamma-\epsilon} K_{i}\right) \text { a.s. }
$$

where we supposed that $a_{n}>0$ (the other case is very similar). Proceeding as for the handling of $\Delta_{i}^{1}\left(\theta_{0}\right)$, and using $\frac{2}{3} x \leq \ln (1+x) \leq x$ for $x \in[0,1 / 2]$, we obtain : for all $\delta>0, \theta \in] 1-\delta, 1+\delta[, \epsilon>0$ and $n$ sufficiently large,

$$
\frac{2}{3}\left(\frac{1-\epsilon}{1+\delta}\right) \gamma U_{i}^{\gamma+\epsilon} K_{i} \leq \frac{\Delta_{i}^{1}(\theta)}{a_{n}} \leq\left(\frac{1+\epsilon}{1-\delta}\right) \gamma U_{i}^{\gamma-\epsilon} K_{i} \text { a.s. }
$$

Since (32) ensures that $\frac{\Delta_{i}^{1}(\theta)}{a_{n}}$ is of constant sign, for $n$ large enough we have

$$
\sup _{\theta \in \mathcal{B}_{n}} \max _{i \leq k_{n}}\left|\Delta_{i}^{1}(\theta)\right| \leq \sqrt{k_{n}} a_{n} \gamma\left(\frac{1+\epsilon}{1-\delta}\right) \frac{\max _{i \leq k_{n}} U_{i}^{\gamma-\epsilon} K_{i}}{\sqrt{k_{n}}} \text { a.s. }
$$


We conclude using Lemmas 3 and 5 and assumption $\sqrt{k_{n}} a_{n} \rightarrow 0$. The proof for $\Delta_{i}^{2}(\theta)$ is very similar.

\subsubsection{Proof of (14) and (16)}

Recall that $A_{n}(\theta)=\frac{1}{k_{n}} \sum_{i=1}^{k_{n}} \frac{\partial g_{0}}{\partial \theta}\left(Z_{i, n}, \theta\right)$ and let $A_{n}^{*}(\theta):=\frac{1}{k_{n}} \sum_{i=1}^{k_{n}} \frac{\partial g_{0}}{\partial \theta}\left(Z_{i}, \theta\right)$, where the $Z_{i}$ were introduced previously. We write

$$
A_{n}(\theta)-A=\left(A_{n}(\theta)-A_{n}^{*}(\theta)\right)+\left(A_{n}^{*}(\theta)-A_{n}^{*}\left(\theta_{0}\right)\right)+\left(A_{n}^{*}\left(\theta_{0}\right)-A\right)
$$

and we will handle separately the three terms on the right hand side above. The third term goes to 0 a.s. according to the strong law of large numbers (SLLN for short) and by definition of the $Z_{i}$ and $A$. The same is true (uniformly in $\theta$ ) for the second term, since part 3 of Lemma 2 implies

$$
\sup _{\theta \in \mathcal{B}_{n}}\left\|A_{n}^{*}(\theta)-A_{n}^{*}\left(\theta_{0}\right)\right\| \leq\left(\sup _{\theta \in \mathcal{B}_{n}}\left\|\theta-\theta_{0}\right\|\right) \frac{1}{k_{n}} \sum_{i=1}^{k_{n}} G_{3}\left(Z_{i}\right) .
$$

and the SLLN applies, thanks to part 4 of Lemma 2. It remains to study the first term of (33) uniformly in $\theta$ in order to prove (14). We have

$$
A_{n}(\theta)-A_{n}^{*}(\theta)=\frac{1}{k_{n}} \sum_{i=1}^{k_{n}} \tilde{\Delta}_{i}(\theta)
$$

where the two components of $\tilde{\Delta}_{i}(\theta)$ are

$$
\begin{aligned}
& \tilde{\Delta}_{i}^{1}(\theta)=-\theta^{-2} \gamma Z_{i, n}\left(1+\gamma Z_{i, n} / \theta\right)^{-1}+\theta^{-2} \gamma Z_{i}\left(1+\gamma Z_{i} / \theta\right)^{-1} \\
& \tilde{\Delta}_{i}^{2}(\theta)=-r \theta^{-2} Z_{i, n}\left(1+\gamma Z_{i, n} / \theta\right)^{r / \gamma-1}+r \theta^{-2} Z_{i}\left(1+\gamma Z_{i} / \theta\right)^{r / \gamma-1} .
\end{aligned}
$$

We shall give details for $\tilde{\Delta}_{i}^{1}(\theta)$ and the case $a_{n}>0$ (the case $a_{n}<0$ and the treatment of $\tilde{\Delta}_{i}^{2}(\theta)$ can be handled very similarly). Let $\left.\delta>0, \theta \in\right] 1-\delta, 1+\delta\left[\right.$, and $V_{i}$ denote $\left(1+\gamma Z_{i} / \theta\right)^{-1}$. Use of the Potter-type bounds (26) leads to the following bounds (for all $\epsilon>0$ and $n$ sufficiently large),

$V_{i}\left(1+a_{n} \theta^{-1}(1+\epsilon) \gamma U_{i}^{-\epsilon} K_{i} V_{i}\right)^{-1} \leq\left(1+\gamma Z_{i, n} / \theta\right)^{-1} \leq V_{i}\left(1+a_{n} \theta^{-1}(1-\epsilon) \gamma U_{i}^{\epsilon} K_{i} V_{i}\right)^{-1}$ a.s.

After multiplication by $-\theta^{-2} \gamma Z_{i, n}$ and another use of (26), we obtain

$$
\begin{aligned}
& -\theta^{-2} \gamma Z_{i} V_{i}\left\{\left(1+a_{n} B_{i}^{-}\right)^{-1}-1\right\}-a_{n} \theta^{-2}(1+\epsilon) \gamma U_{i}^{-\epsilon} K_{i} V_{i}\left(1+a_{n} B_{i}^{-}\right)^{-1} \\
& \quad \leq \tilde{\Delta}_{i}^{1}(\theta) \leq-\theta^{-2} \gamma Z_{i} V_{i}\left\{\left(1+a_{n} B_{i}^{+}\right)^{-1}-1\right\}-a_{n} \theta^{-2}(1-\epsilon) \gamma U_{i}^{\epsilon} K_{i} V_{i}\left(1+a_{n} B_{i}^{+}\right)^{-1}
\end{aligned}
$$

where $B_{i}^{-}=\theta^{-1}(1-\epsilon) \gamma U_{i}^{\epsilon} K_{i} V_{i}$ and $B_{i}^{+}=\theta^{-1}(1+\epsilon) \gamma U_{i}^{-\epsilon} K_{i} V_{i}$. Let us handle the upper bound first. We find easily that $(1-\delta) U_{i}^{\gamma} \leq V_{i} \leq(1+\delta) U_{i}^{\gamma}$, and therefore, by Lemmas 3 and 5 , and assumption $\sqrt{k_{n}} a_{n} \rightarrow 0$,

$$
0 \leq \sup _{|\theta-1| \leq \delta} \max _{i \leq k_{n}}\left(a_{n} B_{i}^{+}\right) \leq(1+\epsilon)(1+\delta)(1-\delta)^{-1} \gamma a_{n} \max _{i \leq k_{n}} U_{i}^{\gamma-\epsilon} K_{i}=o(1) \text { a.s. }
$$

Consequently, using $(1+x)^{-1}-1=-x(1+x)^{-1}$, for $n$ sufficiently large and uniformly in $\theta \in] 1-\delta, 1+\delta$ [, we find (almost surely)

$$
\begin{aligned}
& \frac{1}{k_{n}} \sum_{i=1}^{k_{n}} \frac{\tilde{\Delta}_{i}^{1}(\theta)}{a_{n}} \\
& \leq \frac{(1+\epsilon)(1+\delta)^{2}}{(1-\delta)^{3}} \frac{1}{k_{n}} \sum_{i=1}^{k_{n}}\left(\left(1-U_{i}^{\gamma}\right) U_{i}^{\gamma-\epsilon} K_{i}\right)-\frac{(1-\epsilon)(1-\delta)}{2(1+\delta)^{2}} \gamma \frac{1}{k_{n}} \sum_{i=1}^{k_{n}}\left(U_{i}^{\gamma+\epsilon} K_{i}\right)=O(1)
\end{aligned}
$$


The lower bound can be handled in the same way. Note that (16) is an immediate consequence of (14).

\subsubsection{Proof of (15)}

Recall that $B_{n}(\theta)=k_{n}^{-1} \sum_{i=1}^{k_{n}} g_{0}\left(Z_{i, n}, \theta\right) g_{0}\left(Z_{i, n}, \theta\right)^{t}$ and let

$$
B_{n}^{*}(\theta):=k_{n}^{-1} \sum_{i=1}^{k_{n}} g_{0}\left(Z_{i}, \theta\right) g_{0}\left(Z_{i}, \theta\right)^{t}
$$

where the $Z_{i}$ were introduced previously. We write

$$
B_{n}(\theta)-B=\left(B_{n}(\theta)-B_{n}^{*}(\theta)\right)+\left(B_{n}^{*}(\theta)-B_{n}^{*}\left(\theta_{0}\right)\right)+\left(B_{n}^{*}\left(\theta_{0}\right)-B\right)
$$

The third term in the relation above goes to 0 a.s. according to the SLLN and by definition of the $Z_{i}$ and $B$. Let us deal with the second term. For $\theta \in \mathcal{B}_{n}$, there exists some $\theta_{n}^{*}$ between $\theta_{0}$ and $\theta$ such that (using parts 1 and 2 of Lemma 2)

$$
\begin{aligned}
\left\|B_{n}^{*}(\theta)-B_{n}^{*}\left(\theta_{0}\right)\right\| & \leq\left\|\theta-\theta_{0}\right\| \cdot \frac{2}{k_{n}} \sum_{i=1}^{k_{n}}\left\|\frac{\partial g_{0}}{\partial \theta}\left(Z_{i}, \theta_{n}^{*}\right)\right\|\left\|g_{0}\left(Z_{i}, \theta_{n}^{*}\right)\right\| \\
& \leq k_{n}^{-1 / 3} \max _{i \leq k_{n}}\left(G_{1}\left(Z_{i}\right)\right)^{1 / 3} \frac{2}{k_{n}} \sum_{i=1}^{k_{n}} G_{2}\left(Z_{i}\right) .
\end{aligned}
$$

Therefore, combining part 4 of Lemma 2, Lemma 5 , and the SLLN, we see that $\| B_{n}^{*}(\theta)-$ $B_{n}^{*}\left(\theta_{0}\right) \|$ almost surely goes to 0 as $n \rightarrow \infty$, uniformly in $\theta \in \mathcal{B}_{n}$.

It remains to study the first term of (35) uniformly in $\theta$ in order to prove (15). We have

$$
\begin{aligned}
B_{n}(\theta)-B_{n}^{*}(\theta) & =\frac{1}{k_{n}} \sum_{i=1}^{k_{n}} \Delta_{i}^{t}(\theta) g_{0}\left(Z_{i}, \theta\right)+\frac{1}{k_{n}} \sum_{i=1}^{k_{n}} \Delta_{i}(\theta)\left(g_{0}\left(Z_{i}, \theta\right)\right)^{t}+\frac{1}{k_{n}} \sum_{i=1}^{k_{n}} \Delta_{i}(\theta)\left(\Delta_{i}(\theta)\right)^{t} \\
& =\Gamma_{1, n}^{t}(\theta)+\Gamma_{1, n}(\theta)+\Gamma_{2, n}(\theta)
\end{aligned}
$$

with

$$
\Delta_{i}(\theta)\left(g_{0}\left(Z_{i}, \theta\right)\right)^{t}=\left(\begin{array}{cc}
\Delta_{i}^{1}(\theta)\left(\ln \left(1+\gamma \frac{Z_{i}}{\theta}\right)-\gamma\right) & \Delta_{i}^{1}(\theta)\left(\left(1+\gamma \frac{Z_{i}}{\theta}\right)^{r / \gamma}-\frac{1}{1-r}\right) \\
\Delta_{i}^{2}(\theta)\left(\ln \left(1+\gamma \frac{Z_{i}}{\theta}\right)-\gamma\right) & \Delta_{i}^{2}(\theta)\left(\left(1+\gamma \frac{Z_{i}}{\theta}\right)^{r / \gamma}-\frac{1}{1-r}\right)
\end{array}\right)
$$

and

$$
\Delta_{i}(\theta)\left(\Delta_{i}(\theta)\right)^{t}=\left(\begin{array}{cc}
\left(\Delta_{i}^{1}(\theta)\right)^{2} & \Delta_{i}^{1}(\theta) \Delta_{i}^{2}(\theta) \\
\Delta_{i}^{1}(\theta) \Delta_{i}^{2}(\theta) & \left(\Delta_{i}^{2}(\theta)\right)^{2}
\end{array}\right)
$$

Considering the first element of the matrix $\Gamma_{1, n}(\theta)$, we have

$a_{n}^{-1}\left|\frac{1}{k_{n}} \sum_{i=1}^{k_{n}} \Delta_{i}^{1}(\theta)\left(\ln \left(1+\gamma \frac{Z_{i}}{\theta}\right)-\gamma\right)\right| \leq \sqrt{\frac{1}{k_{n}} \sum_{i=1}^{k_{n}}\left(\frac{\Delta_{i}^{1}(\theta)}{a_{n}}\right)^{2}} \sqrt{\frac{1}{k_{n}} \sum_{i=1}^{k_{n}}\left(\ln \left(1+\gamma \frac{Z_{i}}{\theta}\right)-\gamma\right)^{2}}$

and, applying the Cauchy-Schwarz inequality too for dealing with the other elements of $\Gamma_{1, n}(\theta)$ and $\Gamma_{2, n}(\theta)$, the convergence $B_{n}(\theta)-B_{n}^{*}(\theta) \rightarrow 0$ (uniformly for $\theta \in \mathcal{B}_{n}$ ) will be proved as soon as we show that the means over $i=1$ to $k_{n}$ of each of the following quantities are almost surely bounded uniformly for $\theta \in \mathcal{B}_{n}$ :

$$
\left(\ln \left(1+\gamma \frac{Z_{i}}{\theta}\right)-\gamma\right)^{2},\left(\left(1+\gamma \frac{Z_{i}}{\theta}\right)^{r / \gamma}-\frac{1}{1-r}\right)^{2},\left(\frac{\Delta_{i}^{1}(\theta)}{a_{n}}\right)^{2},\left(\frac{\Delta_{i}^{2}(\theta)}{a_{n}}\right)^{2}, \frac{\Delta_{i}^{1}(\theta) \Delta_{i}^{2}(\theta)}{\left(a_{n}\right)^{2}}
$$


Using Lemma 2, we see that the first two elements of this list are both uniformly bounded by $\frac{1}{k_{n}} \sum_{i=1}^{k_{n}}\left(G_{1}\left(Z_{i}\right)\right)^{2 / 3}$ which converges almost surely. On the other hand, according to relation (32) and since $\mathbb{E}\left(U_{1}^{2 \gamma-2 \epsilon} K_{1}^{2}\right)$ is finite (by Lemma 3 ), $k_{n}^{-1} \sum_{i=1}^{k_{n}}\left(\Delta_{i}^{1}(\theta) / a_{n}\right)^{2}$ is uniformly almost surely bounded. Similarly the same is true for $k_{n}^{-1} \sum_{i=1}^{k_{n}}\left(\Delta_{i}^{2}(\theta) / a_{n}\right)^{2}$, as well as for $k_{n}^{-1} \sum_{i=1}^{k_{n}} \Delta_{i}^{1}(\theta) \Delta_{i}^{2}(\theta) / a_{n}^{2}$, and the proof of (15) is over.

\subsection{Proof of Theorem 1}

We proceed as in the start of the proof of Theorem 2, and consider that the variables $Y_{i}$ are the variables $\bar{F}_{u_{n}}^{-1}\left(U_{i}\right)$ where $\left(U_{i}\right)_{i \geq 1}$ is an i.i.d. sequence of standard uniform variables. Recall that

$$
l(\gamma, \sigma)=2 \sum_{i=1}^{k_{n}} \log \left(1+<\lambda(\gamma, \sigma), g\left(Y_{i}, \gamma, \sigma\right)>\right) .
$$

Defining $Z_{i, n}=Y_{i} / \sigma_{0 n}, \theta_{0}=\left(\gamma_{0}, 1\right), \theta=(\gamma, s)$, and

$$
\tilde{l}(\theta)=2 \sum_{i=1}^{k_{n}} \log \left(1+<\tilde{\lambda}(\theta), g\left(Z_{i, n}, \theta\right)>\right)
$$

where $\tilde{\lambda}(\theta)$ is such that

$$
\frac{1}{k_{n}} \sum_{i=1}^{k_{n}}\left(1+<\tilde{\lambda}(\theta), g\left(Z_{i, n}, \theta\right)>\right)^{-1} g\left(Z_{i, n}, \theta\right)=0,
$$

it comes that $l\left(\gamma_{0}, \sigma_{0 n}\right)=\tilde{l}\left(\theta_{0}\right)$ since $g\left(Z_{i, n}, \gamma, s\right)=g\left(Y_{i}, \gamma, \sigma_{0 n} s\right)$. We thus need to prove that $\tilde{l}\left(\theta_{0}\right)$ converges in distribution to $\chi^{2}(2)$. Following a very classical outline in empirical likelihood theory, it is easy to prove that this convergence is guaranteed as soon as we have the following statements (as $n \rightarrow \infty)$

$$
\begin{gathered}
\frac{1}{k_{n}} \sum_{i=1}^{k_{n}} g\left(Z_{i, n}, \theta_{0}\right) \stackrel{\mathbb{P}}{\longrightarrow} 0, \quad \frac{1}{k_{n}} \sum_{i=1}^{k_{n}} g\left(Z_{i, n}, \theta_{0}\right)\left(g\left(Z_{i, n}, \theta_{0}\right)\right)^{t} \stackrel{\mathbb{P}}{\longrightarrow} B, \\
\frac{1}{\sqrt{k_{n}}} \sum_{i=1}^{k_{n}} g\left(Z_{i, n}, \theta_{0}\right) \stackrel{d}{\longrightarrow} \mathcal{N}(0, B), \quad \max _{i \leq k_{n}}\left\|g\left(Z_{i, n}, \theta_{0}\right)\right\|=O_{\mathbb{P}}\left(\sqrt{k_{n}}\right)
\end{gathered}
$$

However, these statements are included in Proposition 1 and therefore Theorem 1 is proved.

Note : Proposition 1 was stated under the assumption that $r<1 / 3$, but in fact $r<$ $1 / 2$ is sufficient in order to prove all the results concerning $\theta_{0}$ only and not for $\theta$ in a neighborhood of it (and the covariance matrix $B$ is well defined and invertible as soon as $r<1 / 2)$.

\section{Conclusion}

This work deals with the problem of finding confidence regions for the parameters of the approximating GPD distribution in the classical POT framework, for general heavy tailed distributions $(\gamma>0)$. It is shown that the application of the empirical likelihood 
(EL) method to the estimating equations of J. Zhang (2007) yields confidence regions with improved coverage accuracy in comparison to the Wald-type confidence regions issued from the CLT for some estimators of the couple $(\gamma, \sigma)$ (including the maximum likelihood estimator). It is also observed that coverage accuracy is not always as good as one would expect, which means that this subject (and the related one of EL calibration) would need to be further investigated.

A profile EL based confidence interval for the tail index is also obtained, and its performance in terms of coverage probability has been compared to that of the confidence interval (CI) described in J.C. Lu and L. Peng (2002) and L. Peng and Y. Qi (2006) (which is known to perform better than the Wald-type CI based on the CLT for the Hill estimator). In some simulations, the interval of Lu, Peng and Qi shows better performance, but in others this performance is limited to a very short range of number $k_{n}$ of excesses : this instability with respect to $k_{n}$ is much less present for the CI based on Zhang's equations.

We shall finish this conclusion with two remarks. The first is that some of the methodology of the proof of the profile EL result (inspired by Y. S. Qin and J. Lawless (1994)) could prove useful in other settings (Proposition 1 lists properties which yield convergence in distribution of empirical likelihood ratio when the observations form a triangular array). The second remark is that plug-in calibration could be an interesting subject to investigate for obtaining CI for $\gamma$, in particular in order to shorten computation time.

\section{Appendix}

Lemma 2. Let $\gamma>0, r<1 / 3$ and, for $\theta>0$,

$$
g_{0}(z, \theta):=\left(\begin{array}{c}
g_{0}^{1}(z, \theta) \\
g_{0}^{2}(z, \theta)
\end{array}\right)=\left(\begin{array}{c}
\ln (1+\gamma z / \theta)-\gamma \\
(1+\gamma z / \theta)^{r / \gamma}-(1-r)^{-1}
\end{array}\right) .
$$

If we consider, for some positive constants $c_{1}, c_{1}^{\prime}, c_{2}, c_{3}$ depending on $r$ and $\gamma$,

$$
\begin{array}{ccc}
G_{1}(z)= & c_{1}\left(\ln (1+\gamma z)+(1+\gamma z)^{r / \gamma}+c_{1}^{\prime}\right)^{3} \\
G_{2}(z)= & c_{2}\left(z(1+\gamma z)^{-1}+z(1+\gamma z)^{r / \gamma-1}\right) \\
G_{3}(z)= & c_{3}\left(z(1+\gamma z)^{-1}+z(1+\gamma z)^{r / \gamma-1}+z^{2}(1+\gamma z)^{-2}+z^{2}(1+\gamma z)^{r / \gamma-2}\right) .
\end{array}
$$

then there exists a neighborhood of $\theta_{0}=1$ such that for all $\theta$ in this neighborhood and $\forall z \geq 0$,

1. $\left\|g_{0}(z, \theta)\right\|^{3} \leq G_{1}(z)$

2. $\left\|\frac{\partial g_{0}}{\partial \theta}(z, \theta)\right\| \leq G_{2}(z)$

3. $\left\|\frac{\partial^{2} g_{0}}{\partial^{2} \theta}(z, \theta)\right\| \leq G_{3}(z)$

4. If $Z$ has distribution $\operatorname{GPD}(\gamma, 1)$, then $\mathbb{E}\left(G_{j}(Z)\right)$ is finite for each $j \in\{1,2,3\}$.

Proof of Lemma 2: we shall first give details for part 1, since parts 2 and 3 can be treated similarly.

We shall bound from above $\left|g_{0}^{1}(z, \theta)\right|$ and $\left|g_{0}^{2}(z, \theta)\right|$ in the neighborhood of $\theta_{0}=1$. For 
$\delta>0$ and $\theta \in[1-\delta, 1+\delta]$, we have,

$$
\ln \left(1+\frac{\gamma z}{1+\delta}\right)-\gamma \leq g_{0}^{1}(z, \theta) \leq \ln \left(1+\frac{\gamma z}{1-\delta}\right)-\gamma
$$

and if $r<0$ (the case $r>0$ is similar)

$$
\left(1+\frac{\gamma z}{1-\delta}\right)^{r / \gamma}-\frac{1}{1-r} \leq g_{0}^{2}(z, \theta) \leq\left(1+\frac{\gamma z}{1+\delta}\right)^{r / \gamma}-\frac{1}{1-r}
$$

According to Lemma 4 , if we take $\delta<\frac{1}{3}$, we thus have (for some positive constant $c$ )

$$
\left|g_{0}^{1}(z, \theta)\right| \leq \ln (1+\gamma z)+\gamma+\ln (3 / 2), \quad \text { and }\left|g_{0}^{2}(z, \theta)\right| \leq c(1+\gamma z)^{r / \gamma}+\frac{1}{1-r} .
$$

This concludes the proof of part 1 .

If $U$ is uniformly distributed on $[0,1]$, then it is easy to check that the expectations $\mathbb{E}\left[(\ln (1+\gamma Z))^{a}(1+\gamma Z)^{r b / \gamma}\right]=\gamma^{a} \mathbb{E}\left[(-\ln U)^{a} U^{-r b}\right]$ are finite for every $a$ and $b$ in $\{0,1,2,3\}$ because we assumed that $r<1 / 3$. Therefore $\mathbb{E}\left(G_{1}(Z)\right)$ is finite. Similar simple integral calculus leads to the same conclusion for $\mathbb{E}\left(G_{2}(Z)\right)$ and $\mathbb{E}\left(G_{3}(Z)\right)$.

Lemma 3. Let $\gamma>0, \alpha \in \mathbb{R}, \beta \geq 0$ and $U$ a uniform $[0,1]$ random variable .

(i) If $1-\alpha-\gamma>0$, then $\mathbb{E}\left(U^{-\alpha} K_{\gamma, \rho}\left(\frac{1}{U}\right)(-\ln U)^{\beta}\right)$ is finite.

(ii) If $1-\alpha-2 \gamma>0$, then $\mathbb{E}\left(U^{-\alpha} K_{\gamma, \rho}^{2}\left(\frac{1}{U}\right)(-\ln U)^{\beta}\right)$ is finite.

Proof of Lemma 3 : we have

$$
\begin{aligned}
K_{\gamma, \rho}(x) & =\frac{1}{\rho}\left(\frac{x^{\gamma+\rho}}{\gamma+\rho}-\frac{x^{\gamma}}{\gamma}\right)+\frac{1}{\gamma+\rho} & & \text { if } \gamma+\rho \neq 0 \text { and } \rho \neq 0 \\
& =-\frac{1}{\gamma}\left(\ln x-\frac{x^{\gamma}-1}{\gamma}\right) & & \text { if } \gamma+\rho=0 \text { and } \rho \neq 0 \\
& =\frac{1}{\gamma^{2}}\left(x^{\gamma}(\gamma \ln x-1)+1\right) & & \text { if } \rho=0 .
\end{aligned}
$$

We consider statement $(i)$ and provide details only for the case $\gamma+\rho \neq 0$ and $\rho \neq 0$ (all the other cases being handled the same way). A simple change in variables readily gives

$$
\begin{aligned}
\mathbb{E}\left(U^{-\alpha} K_{\gamma, \rho}\left(\frac{1}{U}\right)(-\ln U)^{\beta}\right)= & \frac{1}{\rho(\gamma+\rho)} \int_{0}^{+\infty} \exp ((\alpha+\gamma+\rho-1) y) y^{\beta} d y \\
& -\frac{1}{\rho \gamma} \int_{0}^{+\infty} \exp ((\alpha+\gamma-1) y) y^{\beta} d y \\
& +\frac{1}{\gamma(\gamma+\rho)} \int_{0}^{+\infty} \exp ((\alpha-1) y) y^{\beta} d y .
\end{aligned}
$$

But $\int_{0}^{+\infty} e^{u y} y^{\beta} d y$ being finite if and only if $u<0$, this concludes the proof of $(i)$ since $\gamma$ is positive and $\rho$ is negative, in this case. The proof of statement (ii) involves the same arguments and is thus omitted.

Lemma 4. Let $\gamma>0, z>0$ and $\delta \in[0,1 / 3]$. Then,

$$
\frac{1}{2}<\frac{1+\frac{\gamma z}{1 \pm \delta}}{1+\gamma z}<\frac{3}{2} \quad \text { and } \quad\left|\ln \left(1+\frac{\gamma z}{1 \pm \delta}\right)-\ln (1+\gamma z)\right|<\ln (3 / 2)
$$

Proof of Lemma 4 : since

$$
\frac{1+\frac{\gamma z}{1+\delta}}{1+\gamma z}=1-\frac{\delta}{1+\delta} \frac{\gamma z}{1+\gamma z} \text { and } \frac{1+\frac{\gamma z}{1-\delta}}{1+\gamma z}=1+\frac{\delta}{1-\delta} \frac{\gamma z}{1+\gamma z}
$$


it is clear that the first ratio is between $\frac{1}{2}$ and 1 and the second one between 1 and $\frac{3}{2}$. The second statement comes from

$$
\ln \left(1+\frac{\gamma z}{1+d}\right)-\ln (1+\gamma z)=\ln \left(\frac{1}{1+d}\left(1+\frac{d}{1+\gamma z}\right)\right)
$$

which absolute value is bounded by $\ln (4 / 3)$ for $d=\delta$ and by $\ln (3 / 2)$ for $d=-\delta$, since $\delta$ is assumed to be in $[0,1 / 3]$.

Lemma 5. Let $\left(k_{n}\right)$ be an integer sequence such that $k_{n} \rightarrow+\infty$. If $\left(Z_{i}\right)_{i \geq 1}$ is an i.i.d. sequence of non-negative random variables such that $\mathbb{E}\left(\left|Z_{1}\right|^{p}\right)$ for some $p>0$, then

$$
\max _{i \leq k_{n}}\left|Z_{i}\right|=o\left(k_{n}^{1 / p}\right) \text { almost surely, as } n \rightarrow \infty \text {. }
$$

Proof of Lemma 5 : mimicking the proof of Lemma 11.2 in A.B. Owen (2001), we find that $\max _{i \leq n}\left|Z_{i}\right|=o\left(n^{1 / p}\right)$ almost surely and thus it is also true on the subsequence $\left(k_{n}\right)$, so the lemma is proved.

\section{References}

A. Balkema and L. de Haan (1974). Residual life time at a great age. In Ann. Probab. 2, pages $792-801$.

J. Diebolt, A. Guillou and R. Worms (2003). Asymptotic behaviour of the probability-weighted moments and penultimate approximation. In ESAIM: PESS 7, pages 217-236.

J. Diebolt, A. Guillou and I. Rached (2007). Approximation of the distribution of excesses through a generalized probability-weighted moments method. In J. Statist. Plann. Inference 137, pages 841-857.

L. de Haan (1984) Slow variation and characterization of domain of attraction. In Tiago de Olivera, J. (ed) Statistical extremes and applications, pages 31-48.

J. Hosking and J. Wallis (1987). Parameter and quantile estimation for the generalized Pareto distribution. In Technometrics 29 (3), pages 339-349.

J.C. Lu and L. Peng (2002). Likelihood based confidence intervals for the tail index. In Extremes 5 , pages $337-352$.

A. B. Owen (1988). Empirical likelihood ratio confidence intervals for a single functional. In Biometrika $75(2)$, pages $237-249$.

A. B. Owen (1990). Empirical likelihood ratio confidence regions. In Annals of Statistics 18(1) (1990) 90-120.

A. B. Owen (2001). Empirical Likelihood. Chapman \& Hall

J. Pickands III (1975) Statistical inference using extreme order statistics. In Ann. Stat 3, pages 119-131.

L. Peng and Y. Qi (2006) A new calibration method of constructing empirical likelihood-based confidence intervals for the tail index. In Austr.N. Z. J. Stat. 48(1), pages 59-66.

Y. S. Qin and J. Lawless (1994). Empirical likelihood and general estimating equations. In Annals of Statistics 22(1), pages 300-325.

S.S. Rao (1984). Linear Statistical Inference and its applications. John Wiley \& Sons

J.P. Raoult and R. Worms (2003). Rate of convergence for the generalized Pareto approximation of the excesses. In Adv. Applied Prob. 35 (4), pages 1007-1027.

R. L. Smith (1987). Estimating tails of probability distributions. In Ann. Stat 15, pages 1174-1207.

J. Zhang (2007). Likelihood moment estimation for the generalized Pareto distribution. In Australian \& New Zealand J. Stat 49(1), pages 69-77. 